\title{
A REVISED GENERALIZED F-TEST FOR TESTING THE EQUALITY OF GROUP MEANS UNDER NON-NORMALITY CAUSED BY SKEWNESS
}

\author{
Mustafa CAVUS ${ }^{1}$, Berna YAZICI ${ }^{1}$, and Ahmet SEZER ${ }^{2}$ \\ ${ }^{1}$ Eskisehir Technical University, Department of Statistics, Eskisehir, TURKEY \\ ${ }^{2}$ University of Pittsburgh Medical Center, PA, USA
}

\begin{abstract}
The non-normality may occur in the data due to several reasons such as the presence of the outlier or skewness. It leads to lose the power and fail control Type I error probability of the tests which are used to test the equality of the group means under heteroscedasticity. To overcome this problem, a revised generalized F-test (RGF) is proposed to test the equality of group means under heteroscedasticity in which non-normality is caused by skewness in this study. An extensive Monte-Carlo simulation study is conducted to investigate and compare the performance of the proposed test with non-parametric alternatives under several values of skewness, and different number of groups. The proposed RGF is the best choice in the high level of skewness for $k=3,4,5$. The Kruskal-Wallis test shows better performance than the others in small and moderate sample sizes for $k=6$, and 7 . It is shown that the proposed RGF test is superior than the non-parametric alternatives in the most of the conditions.
\end{abstract}

\section{INTRODUCTION}

Classical F-test $(\mathrm{CF})$ is a powerful procedure in testing the equality of group means when the assumptions hold. If one of the assumptions is violated, the power of the CF test is adversely affected. Alexander-Govern (AG), Generalized F (GF), Parametric Bootstrap (PB), and Welch tests are developed when the assumption of variance homogeneity is violated. When the distributional assumption is violated or the distribution of the data is unknown, non-parametric methods may be

2020 Mathematics Subject Classification. 62J10, 62K99, 62F03.

Keywords. ANOVA, generalized p-value, non-normality, skewed distribution, penalized power, doex.

ఐmustafacavus@eskisehir.edu.tr-Corresponding author; bbabloglu@eskisehir.edu.tr; sezera@upmc.edu

(D) 0000-0002-6172-5449; 0000-0001-9843-7355; 0000-0002-5962-4999. 
more appropriate than their parametric counterparts. Kruskal-Wallis (KW) test is the non-parametric counterpart of the CF test and does not depend on normality assumption. In the case of unequal variances, Brunner et al. (1997) improved the Brunner-Dette-Munk (BDM) test as the heteroscedastic alternative of KW test.

There are numerous articles about the effect of both non-normality and heteroscedasticity on testing the equality of several group means. To reduce the negative effect of non-normality, researchers have proposed to use the robust estimators rather than the usual maximum likelihood (ML) estimators of the sample mean and variance to obtain better control of Type I error probability under non-normality and variance heterogeneity. Luh and Guo (1999) modified the AG trimmed mean test and Welch trimmed mean test with Johnson's normality transformation to deal with non-normality. Keselman et al. (2002) improved the Welch-James heteroscedastic test. Luh and Guo (2005) adopted a trimmed means method in conjunction with Hall's invertible transformation into AG and Welch test. Cribbie et al. (2007) found that the nonparametric procedure proposed by Brunner et al. (1997) provided good Type I error control. Kulinskaya and Dollinger (2007) modified the Welch test with the robust estimators: Huber (1964) proposed two estimators of location and scale, Hampel's M-estimator of location with scale estimated by the median absolute deviation, and the trimmed mean with scale estimated by the Winsorized standard deviation. Cribbie et al. (2012) proposed the modified PB test based on the trimmed mean. Ochuko et al. (2015) modified the AG test using the one-step m-estimator as central tendency measure to obtain a more powerful test under non-normality. Karagoz and Saracbasi (2016) proposed the robust Brown-Forsythe tests based on median/MAD and median/ $Q_{n}$ to test the equality of Weibull distributed group means in the presence of outliers. Ozdemir et al. (2018) proposed the $B_{t j}^{2}$ test based on bootstrapped trimmed means. Yusof et al. (2013) proposed the trimmed F-test combined with the robust scale estimators to overcome the problem of inflating Type I error on testing equality of group means for skewed distributions.

The non-normality may occur in the data due to different reasons such as the presence of outliers or skewness. They may lead to lose power and fail to control Type I error probability of the tests which are used to test the equality of group means under heteroscedasticity. Cavus et al. (2017) modified the GF test to obtain a powerful test for non-normality is caused by outliers. However, it is concluded by the Monte-Carlo simulation study that MGF does not maintain its performance when non-normality caused by skewness. Thus, the primary goal of this article is to propose a revised test for testing the equality of the group means under heteroscedasticity in which non-normality caused by skewness.

The rest of the article is organized as follows: Section 2 provides a literature review about testing the equality of group means. Moreover, the alternative ways in case of assumption violation are described. Section 3 describes the methods that are used to test the equality of group means and the proposed method. An 
extensive Monte-Carlo simulation study, conducted in Section 4, and Section 5, gives some concluding remarks.

\section{Generalized Behrens-Fisher Problem in Case of Non-Normality}

The linear model underlying the ANOVA F test within the context of a one-way independent group design is given in (1).

$$
Y_{i j}=\mu_{i}+\epsilon_{i j}
$$

where $Y_{i j}$ is the dependent variable associated with the $i$ th observation in the $j$ th group for $i=1,2, \ldots, n_{i}$ and $j=1,2, \ldots, k . \mu_{i}$ is the group mean for the $i$ th group, and $\epsilon_{i j}$ is the random error component associated with $Y_{i j}$. The null hypothesis $H_{0}: \mu_{1}=\mu_{2}=\ldots=\mu_{k}$ is tested classical F-test assumed that the $\epsilon_{i j}$ 's are independent, normally distributed and have an equal variance $\sigma^{2}$ for each group of $k$. When the normality assumption does not hold, the non-parametric counterparts of the $\mathrm{CF}$ test are used. In the following subsections, KW and BDM tests, which are the non-parametric counterparts of $\mathrm{CF}$, are introduced.

2.1. Bruner-Dette-Munk (BDM) test. Brunner et al. (1997) proposed the following heterogeneous, rank-based F statistic:

$$
T_{B D M}=\frac{N}{\operatorname{tr}\left(M_{11} V\right)} Q M Q^{\prime}
$$

where $M=I-\frac{1}{J} J, V=N \operatorname{diag}\left(\frac{s_{1}^{2}}{n_{1}}, \frac{s_{2}^{2}}{n_{2}}, \ldots, \frac{s_{j}^{2}}{n_{j}}\right), s_{j}^{2}=\frac{1}{N^{2}\left(n_{j}-1\right)} \sum_{j} R_{i j}-\bar{R}_{j}{ }^{2}$, $Q=\frac{1}{N}\left(\bar{R}_{1}-\frac{1}{2}, \bar{R}_{2}-\frac{1}{2}, \ldots, \bar{R}_{j}-\frac{1}{2}\right)$ and $\bar{R}_{j}=\frac{1}{n_{j}} \sum_{i=1}^{n_{j}} R_{i j}$.

$R_{i j}$ is the rank of $X_{i j}$. The null hypothesis is rejected if $T_{B D M} \geq F_{\alpha, v 1, v 2}$ where $v_{1}=\frac{M_{11}[\operatorname{tr}(V)]^{2}}{\operatorname{tr}(M V M V)}, v 2=\frac{[\operatorname{tr}(V)]^{2}}{\operatorname{tr}\left(V^{2} \Lambda\right)}$ and $\Lambda=\operatorname{diag}\left(\frac{1}{n_{1}-1}, \frac{1}{n_{2}-1}, \ldots, \frac{1}{n_{j}-1}\right)$.

2.2. Kruskal-Wallis (KW) test. The well-known non-parametric counterpart of the CF test is the KW test was proposed by Kruskal and Wallis (1952). For this test, the data is ranked so that the smallest of all observations receives a rank of one, and the largest of all observations receives rank $N$. The test statistic is calculated as

$$
T_{K W}=\frac{12}{N(N+1)} \sum_{j=1}^{k} \frac{R_{j}^{2}}{n_{j}}-3(N+1)
$$

is distributed as $\chi_{k-1}^{2}$, where $n_{j}$ is the sample size for the $j$ th sample, $N$ is the total sample size, and $R_{j}^{2}$ is the sum of the ranks for the $j$ th sample.

Oshima and Algina (1992) showed that the KW test is sensitive to the presence of unequal variances, particularly when the group sizes are unequal. Fagerland and 
Sandvick (2009) indicated that when the observations in each group have different shapes, the KW test may give inaccurate results.

\section{Revised Generalized F-Test (RGF)}

Weerahandi (1995) proposed the Generalized F-test (GF) using the concept of the generalized p-values to test the equality of several normal distributed group means under heteroscedasticity. Yazici and Cavus (2021) discussed the performance the GF test under various conditions. Cavus et al. (2017) proposed the modified GF (MGF) test by replacing the maximum likelihood estimators of the sample mean and variance with Huber (1964)'s M-estimators to overcome non-normality caused by outlier(s). They conducted a Monte-Carlo simulation study to show the performance of the MGF in terms of power and Type I error probability. It was clearly pointed out that MGF outperforms the alternatives in the case of nonnormality caused by outlier(s).

In this paper, the performance of MGF is investigated under heteroscedasticity where non-normality is caused by skewness. However, MGF does not maintain its performance under this case. To solve this problem, MGF is improved by using the Huber (1981) second proposal M-estimators instead of Huber (1964) M-estimators. The difference between these estimators is that the calculation of the scale estimator. Huber (1964) uses the median absolute deviation (MAD) as the scale estimator. However, since the second proposal takes into account the iterative version of MAD, it is used in this study for skewed distributions. The test statistic of the newly proposed test is given in (4).

$$
\begin{aligned}
T_{R G F} & =\frac{T_{N}\left(\bar{X}_{1}^{*}, \bar{X}_{2}^{*}, \ldots, \bar{X}_{k}^{*} ; \sigma_{1}^{2 *}, \sigma_{2}^{2 *}, \ldots, \sigma_{k}^{2 *}\right)}{T_{N}\left(\bar{x}_{1}, \bar{x}_{2}, \ldots, \bar{x}_{k} ; v_{1}^{2} / U_{1}, v_{2}^{2} / u_{2}, \ldots, v_{k}^{2} / U_{k}\right)} \\
& =\frac{\sum_{i=1}^{k}\left(n_{i} / \sigma_{i}^{2 *}\right) \bar{X}_{i}^{2 *}-\left[\sum_{i=1}^{k}\left(n_{i} \bar{X}_{i}^{2 *}\right) / \sigma_{i}^{2 *}\right] / \sum_{i=1}^{k} n_{i} / \sigma_{i}^{2 *}}{\sum_{i=1}^{k}\left(n_{i} U_{i} / v_{i}^{2}\right) \bar{x}_{i}^{2 *}-\left[\sum_{i=1}^{k}\left(n_{i} U_{i} / v_{i}^{2}\right) \bar{x}_{i}^{2 *}\right]^{2} / \sum_{i=1}^{k}\left(n_{i} U_{i} / v_{i}^{2}\right)}
\end{aligned}
$$

where $\bar{x}_{i}^{*}$ and $\sigma_{i}^{2 *}$ 's are the mean and scale estimator of Huber (1981) second proposal, respectively. $U_{1}, U_{2}, \ldots, U_{k}$ are independent random variables with $\chi_{n_{i}-1}^{2}, i=$ $1,2, \ldots, k$. Furthermore, $T_{N}\left(\bar{X}_{1}^{*}, \bar{X}_{2}^{*}, \ldots, \bar{X}_{k}^{*} ; \sigma_{1}^{2 *}, \sigma_{2}^{2 *}, \ldots, \sigma_{k}^{2 *}\right) \chi_{n_{i}-1}^{2}$ independently of $U_{1}, U_{2}, \ldots, U_{k}$. The observed value of $T_{M G F *}$ is defined as the value of MGF at $\left(\bar{X}_{1}^{*}, \bar{X}_{2}^{*}, \ldots, \bar{X}_{k}^{*} ; V_{1}^{2}, V_{2}^{2}, \ldots, V_{k}^{2}\right)=\left(\bar{x}_{1}^{*}, \bar{x}_{2}^{*}, \ldots, \bar{x}_{k}^{*} ; v_{1}^{2}, v_{2}^{2}, \ldots, v_{k}^{2}\right)$, the generalized pvalue is given in the following equation.

$$
p=\#\left(\frac{\chi_{k-1}^{2}}{T_{N}\left(\bar{x}_{1}^{*}, \bar{x}_{2}^{*}, \ldots, \bar{x}_{k}^{*} ; v_{1}^{2} / U_{1}, v_{2}^{2} / u_{2}, \ldots, v_{k}^{2} / U_{k}\right.}>1\right)
$$

The p-value of the RGF test is the Monte-Carlo estimate which is computed with 10000 repetitions. It is the rate of the values which provides the condition given in (5). 


\section{Monte-Carlo Simulation Study}

The performance of the RGF test is investigated under heteroscedasticity where non-normality is caused by skewness over the non-parametric counterparts, KW and BDM test. Moreover, MGF is used as the reference test to show the difference between the performance of the RGF. Skewed Exponential Power Distribution (SEPD) is used to simulate the observations from a wide variety of distributions. We included extreme departures from normality as measured by skewness and kurtosis. Zhu and Zilde (2009)'s version of the SEPD is used as follows:

$$
f(x \mid \mu, \sigma, t, p)=\left\{\begin{array}{lll}
\frac{1}{\sigma} K(p) \exp \left(-\frac{1}{p}\left|\frac{x-\mu}{2 t \sigma}\right|^{p}\right) & \text { if } & x \leq \mu \\
\frac{1}{\sigma} K(p) \exp \left(-\frac{1}{p}\left|\frac{x-\mu}{2(1-t) \sigma}\right|^{p}\right) & \text { if } & x>\mu
\end{array}\right.
$$

where $\mu$ and $\sigma$ are location and scale parameter, $p \geq 0$ is the kurtosis parameter, $t \in[0,1]$ is skewness parameter and $K(p)$ is the normalization constant, $K(p)=$ $1 /\left[2 p^{1 / p} \Gamma(1+1 / p)\right]$. The SEPD corresponds to the Normal distribution when $p=2$ and $t=0.5$.

SEPD departures from normality depending on fixed $t$ and $p$ parameters in the simulation study. The Shapiro-Wilk normality test results depend on $t$ and $n$ presented as percentage (\%) in Table 1 . The results are computed for 10000 samples. SEPD departures from normality when the $n$ increases and $t$ moves away from 0.5 and right-skewed when $t$ is smaller than 0.5 , and left-skewed otherwise. A range between 0.1 and 0.9 for $t$ is used to find out the performance of the tests in case of the non-normality caused by skewness.

Beyond the simulation part of this study, power and Type I error probabilities of the tests are calculated with 10000 replications for the nominal size 0.05 concerning the configuration factors which are sample size, effect size, design type, and skewness. Cavus and Yazici (2020) implemented the doex (ver.1.2) R package is used to compute the p-values of the RGF and MGF test. The values of Type I error probability and power of the tests are tabulated for both balanced and unbalanced designs and different number of groups $(k=3,4,5,6,7)$ in the following subsections.

4.1. The properties of the tests to control the Type I error probability. In this section, Type I error probabilities of the tests are given in Tables 2-6 under different scenarios such as heteroscedasticity where non-normality caused by skewness as well as sample size, design type, and the number of the groups $(k)$. According to the Bradley (1978) liberal criterion, a statistical test is considered robust if the empirical Type I error probability is between 0.025 and 0.075 for a nominal level 0.05 . The value of the Type I error probability of a test is pointed out with " $*$ " which is in this interval.

It is clearly concluded that MGF has a serious problem controlling the Type I error probability when RGF outperforms others for most of the scenarios. Cavus et al. (2017) showed that the MGF is the superior test for heteroscedasticity and 
TABLE 1. The values of skewness and non-normality (\%) of the SEPD with fixed $n$ and $t$ parameters

\begin{tabular}{lrrc}
\hline$t_{i}$ & $n_{i}$ & skewness & non-normality (\%) \\
\hline 0.1 & 10 & 0.467 & 0.159 \\
& 30 & 0.741 & 0.579 \\
& 60 & 0.827 & 0.925 \\
0.2 & 10 & 0.399 & 0.121 \\
& 30 & 0.630 & 0.393 \\
& 60 & 0.705 & 0.737 \\
0.3 & 10 & 0.288 & 0.083 \\
& 30 & 0.462 & 0.208 \\
& 60 & 0.523 & 0.412 \\
0.4 & 10 & 0.151 & 0.058 \\
& 30 & 0.246 & 0.086 \\
& 60 & 0.278 & 0.141 \\
0.5 & 10 & 0.001 & 0.046 \\
& 30 & 0.000 & 0.049 \\
& 60 & 0.000 & 0.050 \\
0.6 & 10 & -0.154 & 0.057 \\
& 30 & -0.252 & 0.089 \\
& 60 & -0.280 & 0.136 \\
0.7 & 10 & -0.289 & 0.081 \\
& 30 & -0.470 & 0.218 \\
& 60 & -0.523 & 0.417 \\
0.8 & 10 & -0.398 & 0.115 \\
& 30 & -0.632 & 0.401 \\
& 60 & -0.706 & 0.738 \\
0.9 & 10 & -0.467 & 0.160 \\
& 30 & -0.739 & 0.575 \\
& 60 & -0.828 & 0.927 \\
\hline & & & \\
& & &
\end{tabular}

non-normality caused by outliers. However, it could not maintain the performance for non-normality caused by skewness in terms of Type I error probability. Since MGF is out of the acceptable interval in almost all cases, it can not be evaluated as a robust test.

Type I error probabilities of the RGF test seem to be very conservative when equal and small sample sizes in case of testing equality of three group means $(k=3)$. However, its Type I error probability is very close to the nominal level for large samples with the high level of skewness. RGF and KW tests outperform others in terms of the controlling Type I error probabilities for $k=3$. KW test is better than RGF in unbalanced designs when the RGF is better in balanced designs. While 
$\mathrm{BDM}$ is keeping its performance for all $k$ 's, and $\mathrm{KW}$ are not keeping in higher values of $k$.

Results indicated that the RGF (for $k=3,4$ ) and $\mathrm{KW}$ (for $k=3,4,5$ ) tests are fairly robust for deviations from the assumption of normality caused by skewness in the case of small sample sizes. For the higher values of $k$, none of the procedures considered in this paper control the Type I error probability.

TABLE 2. Type I error probabilities of the tests for $k=3$

\begin{tabular}{|c|c|c|c|c|c|c|c|c|}
\hline & RGF & MGF & KW & BDM & RGF & MGF & KW & $\mathrm{BDM}$ \\
\hline$t_{i}$ & \multicolumn{4}{|c|}{$n_{i}=(5,10,15)$} & \multicolumn{4}{|c|}{$n_{i}=(10,10,10)$} \\
\hline 0.1 & $0.0549^{*}$ & 0.1428 & $0.0364^{*}$ & 0.0836 & 0.0800 & 0.1672 & 0.0819 & 0.0796 \\
\hline 0.2 & $0.0529 *$ & 0.1326 & $0.0341^{*}$ & 0.0773 & $0.0689 *$ & 0.1553 & $0.0728^{*}$ & $0.0706^{*}$ \\
\hline 0.3 & $0.0485^{*}$ & 0.1186 & $0.0308^{*}$ & $0.0702^{*}$ & $0.0615^{*}$ & 0.1382 & $0.0712^{*}$ & $0.0697^{*}$ \\
\hline 0.4 & $0.0422^{*}$ & 0.1120 & $0.0304 *$ & $0.0629 *$ & $0.0536^{*}$ & 0.1238 & $0.0610^{*}$ & $0.0596 *$ \\
\hline 0.5 & $0.0398^{*}$ & 0.1070 & $0.0265^{*}$ & $0.0552^{*}$ & $0.0463^{*}$ & 0.1121 & $0.0561^{*}$ & $0.0555^{*}$ \\
\hline 0.6 & 0.0409* & 0.1109 & $0.0259^{*}$ & $0.0603^{*}$ & $0.0543^{*}$ & 0.1216 & $0.0612^{*}$ & $0.0606^{*}$ \\
\hline 0.7 & $0.0458^{*}$ & 0.1209 & $0.0299^{*}$ & $0.0694^{*}$ & $0.0617^{*}$ & 0.1372 & $0.0660^{*}$ & $0.0652^{*}$ \\
\hline 0.8 & 0.0501* & 0.1337 & $0.0334^{*}$ & $0.0742 *$ & $0.0672^{*}$ & 0.1542 & $0.0711^{*}$ & $0.0686^{*}$ \\
\hline 0.9 & $0.0545^{*}$ & 0.1414 & $0.0377^{*}$ & 0.0847 & 0.0782 & 0.1685 & 0.0784 & 0.0759 \\
\hline$t_{i}$ & \multicolumn{4}{|c|}{$n_{i}=(15,30,45)$} & \multicolumn{4}{|c|}{$n_{i}=(30,30,30)$} \\
\hline 0.1 & 0.0804 & 0.1078 & $0.0692^{*}$ & 0.1577 & 0.0975 & 0.1274 & 0.1322 & 0.1245 \\
\hline 0.2 & $0.0725^{*}$ & 0.1049 & $0.0572^{*}$ & 0.1322 & 0.0792 & 0.1160 & 0.1116 & 0.1049 \\
\hline 0.3 & $0.0649^{*}$ & 0.0893 & $0.0462^{*}$ & 0.0992 & $0.0696^{*}$ & 0.1013 & 0.0876 & 0.0816 \\
\hline 0.4 & $0.0576^{*}$ & 0.0814 & $0.0310^{*}$ & $0.0710^{*}$ & $0.0593^{*}$ & 0.0831 & $0.0684^{*}$ & $0.0646^{*}$ \\
\hline 0.5 & $0.0526^{*}$ & 0.0778 & $0.0307^{*}$ & $0.0595^{*}$ & $0.0524^{*}$ & 0.0779 & $0.0598^{*}$ & $0.0567^{*}$ \\
\hline 0.6 & $0.0546^{*}$ & 0.0802 & $0.0322^{*}$ & $0.0693^{*}$ & $0.0547^{*}$ & 0.0818 & $0.0668^{*}$ & $0.0626^{*}$ \\
\hline 0.7 & $0.0620^{*}$ & 0.0893 & $0.0421^{*}$ & 0.0944 & $0.0651^{*}$ & 0.0970 & 0.0875 & 0.0815 \\
\hline 0.8 & $0.0703^{*}$ & 0.0978 & $0.0535^{*}$ & 0.1239 & 0.0787 & 0.1150 & 0.1080 & 0.1017 \\
\hline 0.9 & 0.0776 & 0.1025 & $0.0647^{*}$ & 0.1511 & 0.0964 & 0.1292 & 0.1325 & 0.1241 \\
\hline$t_{i}$ & \multicolumn{4}{|c|}{$n_{i}=(30,60,90)$} & \multicolumn{4}{|c|}{$n_{i}=(60,60,60)$} \\
\hline 0.1 & 0.0959 & 0.1077 & 0.1198 & 0.2587 & 0.1054 & 0.1184 & 0.2117 & 0.2003 \\
\hline 0.2 & 0.0804 & 0.0931 & 0.0907 & 0.1998 & 0.0969 & 0.1144 & 0.1704 & 0.1610 \\
\hline 0.3 & $0.0672^{*}$ & 0.0800 & $0.0585^{*}$ & 0.1332 & $0.0744^{*}$ & 0.0922 & 0.1170 & 0.1095 \\
\hline 0.4 & $0.0556^{*}$ & $0.0671^{*}$ & $0.0383^{*}$ & 0.0797 & $0.0635^{*}$ & 0.0770 & 0.0783 & $0.0737^{*}$ \\
\hline 0.5 & $0.0553^{*}$ & $0.0659^{*}$ & $0.0319 *$ & $0.0643^{*}$ & $0.0581^{*}$ & $0.0686^{*}$ & $0.0644^{*}$ & $0.0603^{*}$ \\
\hline 0.6 & $0.0550^{*}$ & $0.0674^{*}$ & $0.0376^{*}$ & 0.0784 & $0.0620^{*}$ & 0.0760 & $0.0738^{*}$ & $0.0709^{*}$ \\
\hline 0.7 & $0.0666^{*}$ & 0.0781 & $0.0567^{*}$ & 0.1296 & $0.0688^{*}$ & 0.0892 & 0.1141 & 0.1060 \\
\hline 0.8 & 0.0777 & 0.0925 & 0.0877 & 0.1969 & 0.0899 & 0.1056 & 0.1662 & 0.1558 \\
\hline 0.9 & 0.0913 & 0.1028 & 0.1212 & 0.2620 & 0.0970 & 0.1136 & 0.2097 & 0.1990 \\
\hline
\end{tabular}


TABLE 3. Type I error probabilities of the tests for $k=4$

\begin{tabular}{|c|c|c|c|c|c|c|c|c|}
\hline & RGF & MGF & KW & $\mathrm{BDM}$ & RGF & MGF & KW & $\mathrm{BDM}$ \\
\hline$t_{i}$ & \multicolumn{4}{|c|}{$n_{i}=(4,8,12,16)$} & \multicolumn{4}{|c|}{$n_{i}=(10,10,10,10)$} \\
\hline 0.1 & $0.0691^{*}$ & 0.1810 & $0.0333^{*}$ & 0.0864 & 0.0973 & 0.2272 & 0.0831 & 0.0783 \\
\hline 0.2 & $0.0626^{*}$ & 0.1714 & $0.0318^{*}$ & 0.0784 & 0.0847 & 0.2078 & 0.0761 & $0.0703^{*}$ \\
\hline 0.3 & $0.0529^{*}$ & 0.1475 & $0.0280^{*}$ & $0.0654^{*}$ & $0.0739 *$ & 0.1805 & $0.0720^{*}$ & $0.0674^{*}$ \\
\hline 0.4 & $0.0438^{*}$ & 0.1375 & $0.0258^{*}$ & $0.0561^{*}$ & $0.0636^{*}$ & 0.1617 & $0.0640^{*}$ & $0.0605^{*}$ \\
\hline 0.5 & $0.0416^{*}$ & 0.1318 & 0.0244 & $0.0542 *$ & $0.0544^{*}$ & 0.1467 & $0.0596^{*}$ & $0.0566^{*}$ \\
\hline 0.6 & $0.0467^{*}$ & 0.1401 & 0.0240 & $0.0592^{*}$ & $0.0627^{*}$ & 0.1585 & $0.0640^{*}$ & $0.0608^{*}$ \\
\hline 0.7 & $0.0540^{*}$ & 0.1599 & $0.0282^{*}$ & $0.0710^{*}$ & 0.0763 & 0.1836 & $0.0739 *$ & $0.0692^{*}$ \\
\hline 0.8 & $0.0600^{*}$ & 0.1716 & $0.0300^{*}$ & 0.0785 & 0.0862 & 0.2055 & 0.0787 & $0.0734^{*}$ \\
\hline 0.9 & $0.0664^{*}$ & 0.1863 & $0.0294^{*}$ & 0.0852 & 0.0998 & 0.2282 & 0.0839 & 0.0779 \\
\hline$t_{i}$ & \multicolumn{4}{|c|}{$n_{i}=(12,24,36,48)$} & \multicolumn{4}{|c|}{$n_{i}=(30,30,30,30)$} \\
\hline 0.1 & 0.0944 & 0.1352 & $0.0707^{*}$ & 0.1850 & 0.1143 & 0.1606 & 0.1470 & 0.1349 \\
\hline 0.2 & 0.0823 & 0.1275 & $0.0521^{*}$ & 0.1466 & 0.0962 & 0.1442 & 0.1281 & 0.1163 \\
\hline 0.3 & $0.0659^{*}$ & 0.1102 & $0.0369^{*}$ & 0.1025 & 0.0798 & 0.1191 & 0.0951 & 0.0854 \\
\hline 0.4 & $0.0544^{*}$ & 0.0894 & $0.0257^{*}$ & $0.0700^{*}$ & $0.0618^{*}$ & 0.0961 & $0.0738^{*}$ & $0.0663^{*}$ \\
\hline 0.5 & $0.0514^{*}$ & 0.0859 & 0.0229 & $0.0573^{*}$ & $0.0578^{*}$ & 0.0905 & $0.0649^{*}$ & $0.0587^{*}$ \\
\hline 0.6 & $0.0602 *$ & 0.0967 & $0.0326^{*}$ & 0.0764 & $0.0616^{*}$ & 0.0950 & $0.0698^{*}$ & $0.0634^{*}$ \\
\hline 0.7 & $0.0695^{*}$ & 0.1075 & $0.0419^{*}$ & 0.1029 & $0.0741^{*}$ & 0.1172 & 0.0914 & 0.0824 \\
\hline 0.8 & 0.0800 & 0.1230 & $0.0529^{*}$ & 0.1454 & 0.0940 & 0.1407 & 0.1197 & 0.1083 \\
\hline 0.9 & 0.0943 & 0.1276 & $0.0662 *$ & 0.1798 & 0.1134 & 0.1633 & 0.1464 & 0.1342 \\
\hline$t_{i}$ & \multicolumn{4}{|c|}{$n_{i}=(24,48,72,96)$} & \multicolumn{4}{|c|}{$n_{i}=(60,60,60,60)$} \\
\hline 0.1 & 0.1036 & 0.1181 & 0.1264 & 0.3220 & 0.1250 & 0.1470 & 0.2469 & 0.2215 \\
\hline 0.2 & 0.0883 & 0.1071 & 0.0910 & 0.2403 & 0.1094 & 0.1361 & 0.1985 & 0.1816 \\
\hline 0.3 & $0.0708^{*}$ & 0.0879 & $0.0548^{*}$ & 0.1517 & 0.0856 & 0.1093 & 0.1342 & 0.1208 \\
\hline 0.4 & $0.0580^{*}$ & 0.0752 & $0.0316^{*}$ & 0.0835 & $0.0684^{*}$ & 0.0871 & 0.0854 & 0.0784 \\
\hline 0.5 & $0.0568^{*}$ & $0.0714^{*}$ & $0.0254^{*}$ & $0.0606^{*}$ & $0.0584^{*}$ & 0.0752 & 0.0649 & $0.0577^{*}$ \\
\hline 0.6 & $0.0625^{*}$ & 0.0785 & $0.0341^{*}$ & 0.0841 & $0.0647^{*}$ & 0.0832 & 0.0814 & $0.0739^{*}$ \\
\hline 0.7 & $0.0713^{*}$ & 0.0936 & $0.0585^{*}$ & 0.1504 & 0.0823 & 0.1066 & 0.1270 & 0.1164 \\
\hline 0.8 & 0.0880 & 0.1088 & 0.0911 & 0.2349 & 0.1055 & 0.1301 & 0.1925 & 0.1760 \\
\hline 0.9 & 0.1085 & 0.1228 & 0.1268 & 0.3159 & 0.1209 & 0.1385 & 0.2472 & 0.2269 \\
\hline
\end{tabular}

$\mathrm{t}$ : skewness parameter of SEPD, RGF: new proposed test, $\left(\sigma_{1}, \sigma_{2}, \sigma_{3}, \sigma_{4}\right)=(0.2,0.4,0.6,0.8)$

Several valuable results are obtained. Despite the BDM is the heteroscedastic alternative of the KW test, the property of KW to control Type I error probability is better than $\mathrm{BDM}$ for balanced designs and $k=3$. For higher values of $k$, the BDM test controls the Type I error probability better than the KW test. KW is not able to control Type I error when the distribution near normal.

4.2. The results of penalized power of the tests. Monte-Carlo simulation studies are used to compare the performance of the tests in terms of power and Type I error probability. However, any comparison of the powers is invalid when Type I error probabilities are different. Cavus et al. (2021) proposed the penalized power 
approach in (7) to compare the power of the tests when Type I error probabilities are different.

$$
\gamma=\frac{1-\beta}{\sqrt{1+\left|1-\frac{\alpha_{i}}{\alpha_{0}}\right|}}
$$

TABLE 4. Type I error probabilities of the tests for $k=5$

\begin{tabular}{|c|c|c|c|c|c|c|c|c|}
\hline & RGF & MGF & KW & $\mathrm{BDM}$ & RGF & MGF & KW & $\mathrm{BDM}$ \\
\hline$t_{i}$ & \multicolumn{4}{|c|}{$n_{i}=(5,8,10,12,15)$} & \multicolumn{4}{|c|}{$n_{i}=(10,10,10,10,10)$} \\
\hline 0.1 & 0.1025 & 0.2525 & $0.0435^{*}$ & 0.0924 & 0.1178 & 0.2841 & 0.0866 & 0.0769 \\
\hline 0.2 & 0.0851 & 0.2308 & $0.0397^{*}$ & 0.0810 & 0.1043 & 0.2544 & 0.0802 & $0.0734^{*}$ \\
\hline 0.3 & $0.0730^{*}$ & 0.2021 & $0.0376^{*}$ & $0.0695^{*}$ & 0.0852 & 0.2232 & $0.0714^{*}$ & $0.0655^{*}$ \\
\hline 0.4 & $0.0589^{*}$ & 0.1776 & $0.0324^{*}$ & $0.0605^{*}$ & $0.0720 *$ & 0.1959 & $0.0682^{*}$ & $0.0615^{*}$ \\
\hline 0.5 & $0.0546^{*}$ & 0.1697 & $0.0299 *$ & $0.0557^{*}$ & $0.0629^{*}$ & 0.1772 & $0.0594^{*}$ & $0.0548^{*}$ \\
\hline 0.6 & $0.0596^{*}$ & 0.1832 & $0.0272^{*}$ & $0.0577^{*}$ & $0.0695^{*}$ & 0.1853 & $0.0629^{*}$ & $0.0577^{*}$ \\
\hline 0.7 & $0.0662^{*}$ & 0.1978 & $0.0288^{*}$ & $0.0645^{*}$ & $0.0834^{*}$ & 0.2230 & $0.0706^{*}$ & $0.0650^{*}$ \\
\hline 0.8 & 0.0816 & 0.2267 & $0.0384^{*}$ & 0.0772 & 0.0995 & 0.2464 & 0.0794 & $0.0714^{*}$ \\
\hline 0.9 & 0.0959 & 0.2517 & $0.0415^{*}$ & 0.0885 & 0.1158 & 0.2758 & 0.0839 & 0.0760 \\
\hline$t_{i}$ & \multicolumn{4}{|c|}{$n_{i}=(15,24,30,36,45)$} & \multicolumn{4}{|c|}{$n_{i}=(30,30,30,30,30)$} \\
\hline 0.1 & 0.1149 & 0.1662 & 0.0918 & 0.1884 & 0.1288 & 0.1896 & 0.1590 & 0.1415 \\
\hline 0.2 & 0.0965 & 0.1481 & $0.0685^{*}$ & 0.1489 & 0.1077 & 0.1680 & 0.1338 & 0.1191 \\
\hline 0.3 & 0.0833 & 0.1338 & $0.0526^{*}$ & 0.1079 & 0.0894 & 0.1390 & 0.1003 & 0.0888 \\
\hline 0.4 & $0.0681^{*}$ & 0.1064 & $0.0364^{*}$ & $0.0721^{*}$ & $0.0680^{*}$ & 0.1100 & 0.0786 & $0.0699 *$ \\
\hline 0.5 & $0.0581^{*}$ & 0.0983 & $0.0327^{*}$ & 0.058 & $0.0599^{*}$ & 0.0994 & $0.0655^{*}$ & $0.0578^{*}$ \\
\hline 0.6 & $0.0586^{*}$ & 0.1006 & $0.0362^{*}$ & 0.06 & $0.0652^{*}$ & 0.1069 & $0.0748^{*}$ & $0.0659^{*}$ \\
\hline 0.7 & 0.0780 & 0.1262 & $0.0538^{*}$ & 0.1064 & 0.0794 & 0.1322 & 0.0941 & 0.0830 \\
\hline 0.8 & 0.0920 & 0.1467 & $0.0716^{*}$ & 0.1488 & 0.1083 & 0.1662 & 0.1326 & 0.1176 \\
\hline 0.9 & 0.1114 & 0.1655 & 0.0909 & 0.1886 & 0.1292 & 0.1889 & 0.1565 & 0.1381 \\
\hline$t_{i}$ & \multicolumn{4}{|c|}{$n_{i}=(30,48,60,72,90)$} & \multicolumn{4}{|c|}{$n_{i}=(60,60,60,60,60)$} \\
\hline 0.1 & 0.1242 & 0.1446 & 0.171 & 0.3440 & 0.1431 & 0.1731 & 0.2819 & 0.2525 \\
\hline 0.2 & 0.1062 & 0.1336 & 0.1256 & 0.2608 & 0.1204 & 0.1545 & 0.2214 & 0.1955 \\
\hline 0.3 & 0.0847 & 0.1088 & $0.0734^{*}$ & 0.1544 & 0.0933 & 0.1201 & 0.1428 & 0.1264 \\
\hline 0.4 & $0.0671^{*}$ & 0.0881 & $0.0454^{*}$ & 0.0862 & $0.0695^{*}$ & 0.0913 & 0.0856 & $0.0725^{*}$ \\
\hline 0.5 & $0.0555^{*}$ & 0.0769 & $0.0364^{*}$ & $0.0606^{*}$ & $0.0615^{*}$ & 0.0784 & $0.0660^{*}$ & $0.0578^{*}$ \\
\hline 0.6 & $0.0668^{*}$ & 0.0870 & $0.0451^{*}$ & 0.0853 & $0.0688^{*}$ & 0.0917 & 0.0859 & $0.0737^{*}$ \\
\hline 0.7 & 0.0819 & 0.1128 & 0.0753 & 0.1556 & 0.0867 & 0.1158 & 0.1382 & 0.1218 \\
\hline 0.8 & 0.1056 & 0.1345 & 0.1268 & 0.2630 & 0.1175 & 0.1500 & 0.2109 & 0.1880 \\
\hline 0.9 & 0.1306 & 0.1455 & 0.1704 & 0.3464 & 0.1368 & 0.1693 & 0.2734 & 0.2451 \\
\hline
\end{tabular}

t: skewness parameter of SEPD, RGF: new proposed test,

$$
\left(\sigma_{1}, \sigma_{2}, \sigma_{3}, \sigma_{4}, \sigma_{5}\right)=(0.2,0.4,0.6,0.8,1.0)
$$


TABLE 5. Type I error probabilities of the tests for $k=6$

\begin{tabular}{|c|c|c|c|c|c|c|c|c|}
\hline & RGF & MGF & KW & $\mathrm{BDM}$ & RGF & MGF & KW & $\mathrm{BDM}$ \\
\hline$t_{i}$ & \multicolumn{4}{|c|}{$n_{i}=(4,6,8,12,14,16)$} & \multicolumn{4}{|c|}{$n_{i}=(10,10,10,10,10,10)$} \\
\hline 0.1 & 0.1038 & 0.2959 & $0.0321^{*}$ & 0.0920 & 0.1419 & 0.3390 & 0.0962 & 0.0848 \\
\hline 0.2 & 0.0922 & 0.2699 & $0.0276^{*}$ & 0.0764 & 0.1190 & 0.2991 & 0.0863 & 0.0772 \\
\hline 0.3 & $0.0726^{*}$ & 0.2406 & 0.0235 & $0.0650^{*}$ & 0.0957 & 0.2566 & $0.0725^{*}$ & $0.0640 *$ \\
\hline 0.4 & $0.0616^{*}$ & 0.2202 & 0.0220 & $0.0536^{*}$ & 0.0775 & 0.2255 & $0.0651^{*}$ & $0.0579 *$ \\
\hline 0.5 & $0.0574^{*}$ & 0.2034 & 0.0229 & $0.0514^{*}$ & $0.0661^{*}$ & 0.2073 & $0.0596^{*}$ & $0.0530 *$ \\
\hline 0.6 & $0.0612^{*}$ & 0.2115 & 0.0237 & $0.0551^{*}$ & 0.0791 & 0.2190 & $0.0630^{*}$ & $0.0575 *$ \\
\hline 0.7 & 0.0806 & 0.2376 & $0.0288^{*}$ & $0.0689^{*}$ & 0.0962 & 0.2588 & $0.0730^{*}$ & $0.0656^{*}$ \\
\hline 0.8 & 0.0930 & 0.2680 & $0.0350^{*}$ & 0.0839 & 0.1179 & 0.2916 & 0.0835 & $0.0731^{*}$ \\
\hline 0.9 & 0.1089 & 0.2931 & $0.0355^{*}$ & 0.0957 & 0.1395 & 0.3259 & 0.0926 & 0.0823 \\
\hline$t_{i}$ & \multicolumn{4}{|c|}{$n_{i}=(12,18,24,36,42,48)$} & \multicolumn{4}{|c|}{$n_{i}=(30,30,30,30,30,30)$} \\
\hline 0.1 & 0.1244 & 0.1911 & 0.0783 & 0.2213 & 0.1452 & 0.2211 & 0.1732 & 0.1505 \\
\hline 0.2 & 0.1085 & 0.1762 & $0.0617^{*}$ & 0.1 & & 0.189 & & 0.1203 \\
\hline 0.3 & 0.0844 & 0.1473 & $0.0437^{*}$ & & & 0.1551 & 0.10 & 0.0884 \\
\hline 0.4 & $0.0648^{*}$ & & $0.0283^{*}$ & & & 0.1212 & 9 & $0.0683^{*}$ \\
\hline 0.5 & $0.0579^{*}$ & 0.1 & 0.0244 & 0.05 & $0.0636^{*}$ & 0.1083 & $0.0686^{*}$ & $0.0595^{*}$ \\
\hline 0.6 & $0.0614^{*}$ & 0.1145 & $0.0289^{*}$ & $0.0694^{*}$ & $0.0704^{*}$ & 0.1191 & 0.0792 & $0.0663^{*}$ \\
\hline 0.7 & 0.0826 & 0.1411 & $0.0451^{*}$ & 0.1117 & 0.0873 & 0.1479 & 0.0997 & 0.0862 \\
\hline 0.8 & 0.1022 & 0.1677 & $0.0597^{*}$ & 0.1673 & 0.1185 & 0.1874 & 0.1380 & 0.1203 \\
\hline 0.9 & 0.1229 & 0.1907 & 0.0760 & 0.2220 & 0.1446 & 0.2147 & 0.1718 & 0.1472 \\
\hline$t_{i}$ & \multicolumn{4}{|c|}{$n_{i}=(24,36,48,72,84,96)$} & \multicolumn{4}{|c|}{$n_{i}=(60,60,60,60,60,60)$} \\
\hline 0.1 & 0.1392 & 0.1681 & 0.10 & 0.398 & 1608 & 56 & 0.0 & 0.2722 \\
\hline 0.2 & 0.1161 & 0.1503 & 0.1064 & 0.2 & 0.1319 & 0.1697 & 0.2356 & 0.2032 \\
\hline 0.3 & 0.0855 & & $0.0616^{*}$ & 0.17 & 0.1014 & 0.1331 & 0.1534 & 0.1337 \\
\hline 0.4 & $0.0637^{*}$ & & $0.0321^{*}$ & & $0.0723^{*}$ & 0.0950 & & 0.0756 \\
\hline 0.5 & $0.0555^{*}$ & 0.0798 & 0.0245 & $0.0583^{*}$ & $0.0640^{*}$ & 0.0825 & $0.0679^{*}$ & $0.0546^{*}$ \\
\hline 0.6 & $0.0637^{*}$ & 0.0899 & $0.0333^{*}$ & 0.0866 & 0.0699* & 0.0947 & 0.0862 & $0.0727^{*}$ \\
\hline 0.7 & 0.0872 & 0.1176 & $0.0621^{*}$ & 0.1761 & 0.0915 & 0.1278 & 0.1443 & 0.1239 \\
\hline 0.8 & 0.1129 & 0.1495 & 0.1046 & 0.2997 & 0.1300 & 0.1668 & 0.2314 & 0.2038 \\
\hline 0.9 & 0.1396 & 0.1685 & 0.1500 & 0.3997 & 0.1584 & 0.1923 & 0.3026 & 0.2680 \\
\hline
\end{tabular}


TABLE 6. Type I error probabilities of the tests for $k=7$

\begin{tabular}{|c|c|c|c|c|c|c|c|c|}
\hline & RGF & MGF & KW & $\mathrm{BDM}$ & RGF & MGF & $\mathrm{KW}$ & BDM \\
\hline$t_{i}$ & \multicolumn{4}{|c|}{$n_{i}=(4,6,8,10,12,14,16)$} & \multicolumn{4}{|c|}{$n_{i}=(10,10,10,10,10,10,10)$} \\
\hline 0.1 & 0.1218 & 0.3421 & $0.0356^{*}$ & 0.0935 & 0.1597 & 0.3814 & 0.0985 & 0.0840 \\
\hline 0.2 & 0.1077 & 0.3190 & $0.0341^{*}$ & 0.0855 & 0.1349 & 0.3409 & 0.0854 & 0.0765 \\
\hline 0.3 & 0.0860 & 0.2743 & $0.0276^{*}$ & $0.0661^{*}$ & 0.1066 & 0.2914 & $0.0739 *$ & $0.0643^{*}$ \\
\hline 0.4 & $0.0704^{*}$ & 0.2507 & 0.0234 & $0.0562 *$ & 0.0840 & 0.2552 & $0.0647^{*}$ & $0.0558 *$ \\
\hline 0.5 & $0.0670^{*}$ & 0.2281 & 0.0226 & $0.0526^{*}$ & 0.0724 & 0.2327 & $0.0596^{*}$ & $0.0519^{*}$ \\
\hline 0.6 & $0.0699^{*}$ & 0.2348 & $0.0256^{*}$ & $0.0574^{*}$ & 0.0855 & 0.2499 & $0.0661^{*}$ & $0.0586^{*}$ \\
\hline 0.7 & 0.0852 & 0.2710 & $0.0304^{*}$ & $0.0675^{*}$ & 0.1113 & 0.2981 & $0.0747^{*}$ & $0.0654^{*}$ \\
\hline 0.8 & 0.1037 & 0.3079 & $0.0319^{*}$ & 0.0804 & 0.1359 & 0.3441 & 0.0895 & 0.0786 \\
\hline 0.9 & 0.1229 & 0.3459 & $0.0366^{*}$ & 0.0984 & 0.1606 & 0.3750 & 0.0994 & 0.0870 \\
\hline$t_{i}$ & \multicolumn{4}{|c|}{$n_{i}=(12,18,24,30,36,42,48)$} & \multicolumn{4}{|c|}{$n_{i}=(30,30,30,30,30,30,30)$} \\
\hline 0.1 & 0.1396 & 0.2132 & 0.0849 & 0.2290 & 0.1599 & 0.2429 & 0.1791 & 0.1518 \\
\hline 0.2 & 0.1194 & 0.1920 & $0.0642^{*}$ & 0.1753 & 0.1274 & 0.2097 & 0.1435 & 0.1234 \\
\hline 0.3 & 0.0930 & 0.1635 & $0.0436^{*}$ & 0.1168 & 0.1030 & 0.1698 & 0.1066 & 0.0905 \\
\hline 0.4 & $0.0717^{*}$ & 0.1277 & $0.0306^{*}$ & $0.0696^{*}$ & 0.0779 & 0.1333 & 0.0793 & $0.0668^{*}$ \\
\hline 0.5 & $0.0627^{*}$ & 0.1117 & 0.0244 & $0.0540 *$ & $0.0632^{*}$ & 0.1161 & $0.0686^{*}$ & $0.0577^{*}$ \\
\hline 0.6 & $0.0677^{*}$ & 0.1232 & $0.0280^{*}$ & $0.0697^{*}$ & $0.0746^{*}$ & 0.1302 & 0.0782 & $0.0669^{*}$ \\
\hline 0.7 & 0.0880 & 0.1578 & $0.0430 *$ & 0.1194 & 0.0960 & 0.1673 & 0.1046 & 0.0882 \\
\hline 0.8 & 0.1132 & 0.1886 & $0.0628^{*}$ & 0.1764 & 0.1293 & 0.2119 & 0.1437 & 0.1213 \\
\hline 0.9 & 0.1358 & 0.2072 & 0.0793 & 0.2276 & 0.1580 & 0.2391 & 0.1820 & 0.1563 \\
\hline$t_{i}$ & \multicolumn{4}{|c|}{$n_{i}=(24,36,48,60,72,84,96)$} & \multicolumn{4}{|c|}{$n_{i}=(60,60,60,60,60,60,60)$} \\
\hline 0.1 & 0.1600 & 0.1890 & 0.1759 & 0.4405 & 0.1804 & 0.2197 & 0.3376 & 0.2961 \\
\hline 0.2 & 0.1238 & 0.1606 & 0.1185 & 0.3218 & 0.1466 & 0.1934 & 0.2578 & 0.2237 \\
\hline 0.3 & 0.0923 & 0.1312 & $0.0659 *$ & 0.1859 & 0.1058 & 0.1477 & 0.1660 & 0.1430 \\
\hline 0.4 & $0.0722^{*}$ & 0.1019 & $0.0370^{*}$ & 0.0898 & 0.0762 & 0.1040 & 0.0907 & 0.0766 \\
\hline 0.5 & $0.0612^{*}$ & 0.0885 & $0.0267^{*}$ & $0.0566^{*}$ & $0.0658^{*}$ & 0.0867 & $0.0682^{*}$ & $0.0537^{*}$ \\
\hline 0.6 & $0.0657^{*}$ & 0.0954 & $0.0369 *$ & 0.0873 & $0.0719 *$ & 0.0997 & 0.0848 & $0.0704^{*}$ \\
\hline 0.7 & 0.0875 & 0.1260 & $0.0668^{*}$ & 0.1818 & 0.0981 & 0.1396 & 0.1506 & 0.1278 \\
\hline 0.8 & 0.1242 & 0.1621 & 0.1255 & 0.3208 & 0.1418 & 0.1862 & 0.2552 & 0.2217 \\
\hline 0.9 & 0.1569 & 0.1872 & 0.1780 & 0.4419 & 0.1768 & 0.2159 & 0.3295 & 0.2853 \\
\hline
\end{tabular}

where $\beta$ is Type II error rate, $\alpha_{i}$ is Type I error of the test and $\alpha_{0}$ is the nominal level. Penalized power adjusts the power function with the square root of the percentile deviation between Type I error probability and the nominal level. Thus, penalized power is used to compare the power of the tests in the simulation studies.

Penalized power of the tests are given in Tables 7-11 under different scenarios such as sample size, design type and the number of the groups $(k)$. Indeed a test can be evaluated as an acceptable test when Type I error probability of the test is near to the nominal level and its power as high as possible according to Neyman-Pearson theory. Thus, it is clearly seen that MGF is more powerful than 
the others. However, MGF can not control Type I error probability so that it can not be considered an acceptable test. The second powerful test is RGF especially for small sample size and $k$ 's. Also, it performs better in the unbalanced over the balanced designs. However, its power, like the others, is adversely affected by the increasing number of groups. The power of $\mathrm{KW}$ and BDM tests is least affected by skewness. There are cases where the BDM test performs better than the others in terms of power for small sample sizes. The power of the KW test is the lowest one for unbalanced designs with small sample sizes. However, it is seen that KW is more powerful than RGF especially for balanced designs when the sample size is increased.

From the results in Tables $7-11, \mathrm{KW}$ and BDM tests have similar power when the skewness level is high $(t \sim 0.1$ or 0.9$)$ for $k=3,4$. However, BDM is more powerful than $\mathrm{KW}$ in small sample sizes when the distribution is nearly normal. $(t \sim 0.5)$. Moreover, BDM is more powerful than $\mathrm{KW}$ for unbalanced designs for $k=5,6,7$ whereas $\mathrm{KW}$ is more powerful for balanced designs.

\section{Conclusions}

There are many modifications proposed to the parametric tests instead of using non-parametric tests in case of non-normality and heteroscedasticity. Cavus et al. (2017) proposed a modification to the GF test under non-normality caused by outliers replacing ML estimators of the sample mean and variance with robust estimators. It was shown that the MGF test is powerful than the alternatives in a Monte-Carlo simulation study. Also, the effectiveness of MGF was illustrated by an example of analyzing regional export data by Cavus et al. (2018a). However, it does not maintain the performance under non-normality caused by skewness.

In this study, MGF is revised by using the advantage of the iterative formed scale estimator of Huber (1981) second proposal for non-normality caused by skewness. The performance of the newly proposed RGF test is investigated in an extensive Monte-Carlo simulation study in terms of Type I error probability and penalized power of the test. According to the results which are given in the previous subsections, RGF is superior when non-normality caused by skewness, especially for lower $k$ 's and unbalanced designs. It is more powerful than KW and a better test to control Type I error probability, while the BDM test may be useful for higher $k$ 's. However, BDM can not control Type I error probability in case of high values of skewness.

As a result, we suggest researchers use RGF test for $k=3,4,5$. Because of the decreasing performance of RGF for $k=6,7$, the KW test may be more appropriate to use for the high level of skewness in small and moderate sample sizes-unbalanced designs. However, BDM test should be used for slightly skewed normal distributions $(t \sim 0.5)$ in small and moderate sample sizes-balanced designs. It is concluded and advised that RGF is the best choice in the high level of skewness because of its better performance to control Type I error probability. 
Table 7. Penalized power of the tests for $k=3$

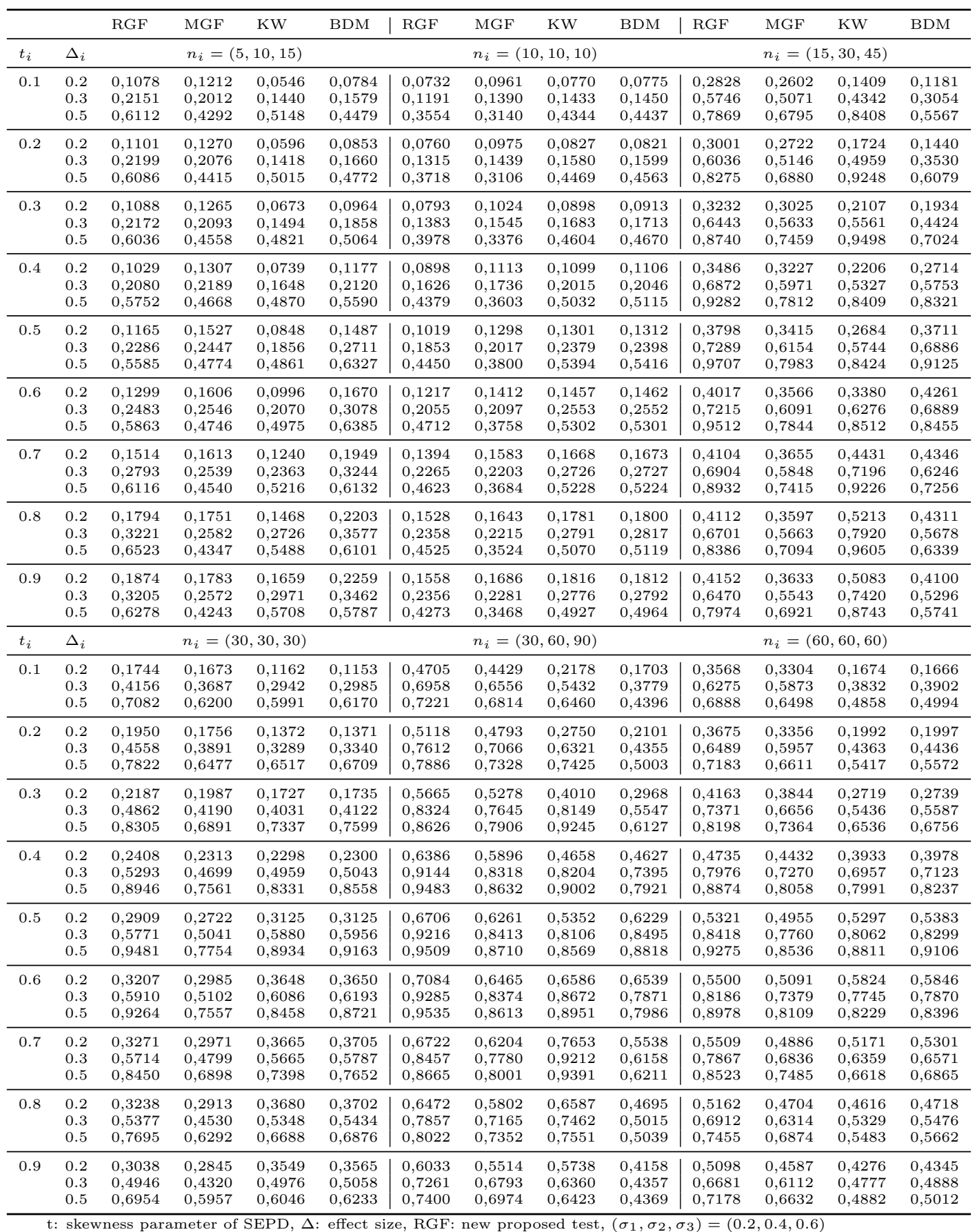


TABLE 8. Penalized power of the tests for $k=4$

\begin{tabular}{|c|c|c|c|c|c|c|c|c|c|c|c|c|c|}
\hline & & GF & IGF & KW & BDM & GF & MGF & KW & BDM & RGF & MGF & KW & BDM \\
\hline$t_{i}$ & $\Delta_{i}$ & \multicolumn{4}{|c|}{$n_{i}=(4,8,12,16)$} & \multicolumn{4}{|c|}{$n_{i}=(10,10,10,10)$} & \multicolumn{4}{|c|}{$n_{i}=(12,24,36,48)$} \\
\hline \multirow[t]{3}{*}{0.1} & 0.2 & 0789 & 0,1112 & 0,0360 & 0,0566 & 418 & 0,1696 & 0,0654 & 0,0560 & 0,1556 & 0,1550 & 0,0737 & 0,0734 \\
\hline & 0.3 & 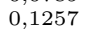 & 47 & 0,0743 & 0,0873 & 541 & 0,1913 & 0,0967 & 0,0809 & 0,3288 & 0,2937 & 0,2099 & 0,1545 \\
\hline & 0.5 & 0,3244 & 0,2792 & 0,2677 & 0,2377 & 0,1116 & 0,2931 & 0,2309 & 0,1991 & 0,6802 & 0,5720 & 0,7035 & 0,4335 \\
\hline \multirow[t]{3}{*}{0.2} & 0.2 & 0,0758 & 0,1075 & 0,0372 & 0,0628 & 0417 & 0,1645 & 0,0713 & 0,0571 & 0,1559 & 0,1574 & 0,0907 & 0,0829 \\
\hline & 0.3 & c & 1420 & 0,0740 & 0,0977 & 543 & 0,1894 & 1012 & 0813 & 495 & 0,3011 & 0,2643 & 0,1800 \\
\hline & 0.5 & 0,3201 & 0,2785 & 0,2618 & 0,2500 & 0,1112 & 0,3036 & 0,2410 & 0,1973 & 7257 & 0,5876 & 0,8117 & 0,4874 \\
\hline \multirow[t]{3}{*}{0.3} & 0.2 & 0,0760 & 1072 & 0,0405 & 0,0701 & 0439 & 0,1553 & 0,0696 & 0,0557 & 0,1798 & 0,1701 & 0,0993 & 0,1106 \\
\hline & 0.3 & 262 & 4 & 7785 & 1110 & 569 & 0,1 & 1063 & 0846 & 3829 & 0,3298 & 0,2656 &, 2354 \\
\hline & 0.5 & 0,3430 & 0,2950 & 0,2575 & 0,2861 & 1163 & 0,3105 & 0,2556 & 0,2048 & 8042 & 0,6255 & 0,7529 & 0,5960 \\
\hline \multirow[t]{3}{*}{0.4} & 0.2 & 0768 & 0,1151 & 0479 & 0,0808 & 0420 & 0,1596 & 0782 & 0,0584 & 0,2055 & 0,2003 & 0,1124 & 0,1560 \\
\hline & 0.3 & & & 18 & 1312 & 591 & & 1216 & & & & & \\
\hline & 0.5 & 0,3375 & 0,3079 & 0,2605 & 0,3397 & 0,1350 & 0,3437 & 0,2900 & 0,2227 & 0,8784 & 0,6939 & 0,7043 & 0,7407 \\
\hline \multirow[t]{3}{*}{0.5} & 0.2 & 94 & 0,1208 & 6 & 0 & 73 & 6 & 0912 & 9 & 2 & 1 & 95 & 281 \\
\hline & 0.3 & & & & & & & & & & & & \\
\hline & 0.5 & 0,3372 & 0,3157 & 0,2754 & 0,3800 & 0,1481 & 0,3686 & 0,3107 & 0,2300 & 0,9048 & 0,7051 & 0,7116 & 0,8521 \\
\hline \multirow[t]{3}{*}{0.6} & 0.2 & c & 0,1234 & 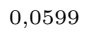 & & 1 & 7 & 4 & 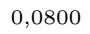 & & 0,2259 & & \\
\hline & 0. & & & & & & & & & & & & \\
\hline & 0.5 & 0,3775 & 0,3110 & 0,2948 & 0,4145 & 0,1636 & 0,3804 & 0,3294 & 0,2514 & 0,8339 & 0,6595 & 0,7803 & 0,7601 \\
\hline \multirow[t]{3}{*}{0.7} & 0.2 & & & & & & & & & & & & 014 \\
\hline & 0. & & & & & & & & & & & & \\
\hline & 0.5 & 0,4022 & 0,3057 & 0,3373 & 0,4228 & 0,1675 & 0,3878 & 0,3417 & 0,2787 & 0,7793 & 0,6246 & 0,8579 & 0,6689 \\
\hline \multirow[t]{3}{*}{0.8} & 0.2 & & 1392 & & & & & & & & & & 14 \\
\hline & 0 & & & & & & & & & & & & \\
\hline & 0.5 & 0,3986 & 0,3021 & 0,3594 & 0,4362 & 0,1709 & 0,4016 & 0,3480 & 0,2909 & 0,7267 & 0,5845 & 0,9108 & 0,5666 \\
\hline \multirow[t]{3}{*}{0.9} & 0.2 & 0 & 14 & 0 & 1623 & 01 & 2463 & 0,1421 & & 2 & 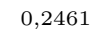 & 4 & 31 \\
\hline & 0. & & & & & & & & & & & & \\
\hline & 0.5 & & 0,3022 & 0,3831 & & & 0,4091 & 0,3596 & & & 0,5769 & 0,8235 &, 5146 \\
\hline \multirow{4}{*}{$\frac{t_{i}}{0.1}$} & $\Delta_{i}$ & \multicolumn{4}{|c|}{$n_{i}=(30,30,30,30)$} & & $\imath_{i}=(24$ & $48,72,96)$ & & & $n_{i}=(60$, & $60,60,60)$ & \\
\hline & 0. & & & & & & & & & & & & 7 \\
\hline & 0 & & & & & & & & & & & & \\
\hline & 0.5 & 0,4392 & 0,4653 & 0,4532 & 0,5160 & 0,6943 & 0,6503 & 0,6245 & 0,3913 & 5782 & 0,4458 & 0,4583 & 0,6784 \\
\hline 0.2 & 0. & & & 0,096 & 0 & & & & & & & 9 & 70 \\
\hline & & & & & & & & & & & & & \\
\hline & 0.5 & 0,4584 & 0,4964 & 0,4891 & 0,5152 & 0,7523 & 0,6831 & 0,7364 & 0,4534 & 0,5979 & 0,4955 & 0,5061 & 0,6809 \\
\hline 0.3 & 0. & 95 & 0 , & 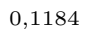 & 0 , & 314 & 171 & 0,2174 & 560 & 0 & ? & 3 & 598 \\
\hline & 0 & & & & & & & & & & & & \\
\hline & 0.5 & 0,4888 & 0,5677 & 0,5788 & 0,5261 & 0,8404 & 0,7541 & 0,9502 & 0,5708 & 0,6638 & 0,6000 & 0,6244 & 0,6833 \\
\hline 0.4 & 0. & 10 & 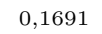 & ( & 1174 & 3892 & 0,3599 & 0,2420 & 0,2546 & 1 & 0,2410 & 6 & 26 \\
\hline & & & & & & & & & & & & & \\
\hline & 0.5 & 0,5463 & 0,6409 & 0,6786 & 0,5452 & 0,9279 & 0,8151 & 0,8519 & 0,7717 & 0,7454 & 0,7519 & 0,7801 & 0,6899 \\
\hline 0.5 & 0. & & & & & & & & & & & & 0,2537 \\
\hline & & & & & & & & & & & & & \\
\hline & 0.5 & 0,5639 & 0,6823 & 0,7500 & 0,5657 & 0,9369 & 0,8357 & 0,8170 & 0,9073 & 0,7962 & 0,8575 & 0,9154 & 0,6937 \\
\hline 0.6 & 0. & & & & & & & & & & & & \\
\hline & & & & & & & & & & & & & \\
\hline & 0.5 & 0,5577 & 0,6683 & 0,7520 & 0,5889 & 0,8930 & 0,7971 & 0,8698 & 0,7709 & 0,7549 & 0,7620 & 0,8125 & 0,6971 \\
\hline 0.7 & & & & & & & & & & & & & \\
\hline & & & & & & & & & & & & & \\
\hline & 0.5 & 0,5091 & 0,5886 & 0,6777 & 0,6067 & 0,8357 & 0,7285 & 0,9238 & 0,5765 & 0,6690 & 0,6100 & 0,6487 & 0,6990 \\
\hline 0.8 & & & & & & & & & & & & & \\
\hline & & & & & & & & & & & & & \\
\hline & 0.5 & 0,4701 & 0,5186 & 0,6031 & 0,6187 & 0,7527 & 0,6760 & 0,7405 & 0,4613 & 0,6053 & 0,4955 & 0,5294 & 0,7013 \\
\hline 0.9 & & & & & & & & & & & & & \\
\hline & 0. & & & & & & & & & & & & \\
\hline & 0.5 & 0,4451 & 0,4728 & 0,5500 & 0,6289 & 0,6780 & 0,6367 & 0,6277 & 0,3978 & 0,5897 & 0,4386 & 0,4675 & 0,7036 \\
\hline
\end{tabular}

$\left(\sigma_{1}, \sigma_{2}, \sigma_{3}, \sigma_{4}, \sigma_{5}\right)=(0.2,0.4,0.6,0.8,1.0)$ 
TABle 9. Penalized power of the tests for $k=5$

\begin{tabular}{|c|c|c|c|c|c|c|c|c|c|c|c|c|c|}
\hline & & RGF & MGF & KW & 3DM & RGF & MGF & $\mathrm{KW}$ & BDM & RGF & MGF & $\mathrm{KW}$ & BDM \\
\hline$t_{i}$ & $\Delta_{i}$ & \multicolumn{4}{|c|}{$n_{i}=(5,8,10,12,15)$} & \multicolumn{4}{|c|}{$n_{i}=(10,10,10,10,10)$} & \multicolumn{4}{|c|}{$n_{i}=(15,24,30,36,45)$} \\
\hline \multirow[t]{3}{*}{0.1} & 0.2 & 5717 & 0,1137 & 0,0342 & 0,0537 & 49 & 0,2050 & 0,0656 & 0,0518 & 0,1053 & 0,1140 & 0,0567 & 0,0756 \\
\hline & 0.3 & 0912 & 1282 & 0547 & 0,0681 & & 0,2147 & & & 0,1909 & & 0,1204 & 0,1112 \\
\hline & 0.5 & 0,1710 & 0,1830 & 0,1594 & 0,1449 & 0,0740 & 0,2626 & 0,1521 & 0,1253 & 0,4701 & 0,4027 & 0,4151 & 0,2958 \\
\hline \multirow[t]{3}{*}{0.2} & 0.2 & 0,0719 & 0,1118 & 0,0368 & 0,0574 & 0,0450 & 0,1958 & 0,0662 & 0,0516 & 0,1083 & 0,1161 & 0,0677 & 0,0727 \\
\hline & 0.3 & 970 & 3 & 0570 & 0751 & 504 & 081 & & 0,0649 & 0,2000 & & 0,1435 & 0,1200 \\
\hline & 0.5 & 0,1815 & 0,1892 & 0,1677 & 0,1644 & 0,0763 & 0,2678 & 0,1543 & 0,1243 & 0,5023 & 0,4231 & 0,4755 & 0,3278 \\
\hline \multirow[t]{3}{*}{0.3} & 0.2 & 0,0698 & 0,1062 & 0,0406 & 0,0626 & 0,0405 & 0,1831 & 0,0671 & 0,0486 & 0,1133 & 0,1176 & 0,0778 & 0,0806 \\
\hline & 0.3 & 889 & 6 & 6641 & 0,0806 & 475 & & 0,0860 & 0,0643 & 0,2117 & 65 & 0,1769 &, 1458 \\
\hline & 0.5 & 0,1889 & 0,1938 & 0,1704 & 0,1790 & 0,0775 & 0,2775 & 0,1761 & 0,1352 & 0,5349 & 0,4458 & 0,5715 & 0,4067 \\
\hline \multirow[t]{3}{*}{0.4} & 0.2 & 5774 & 1070 & 0,0461 & 0,0695 & 0,0395 & 0,1726 & 0,0692 & 0,0505 & 0,1244 &, 1307 & 0,0825 & 0,1053 \\
\hline & 0.3 & 095 & 7 & 22 & 0,1013 & 56 & 1976 & 0,0986 & 0723 & 0,2386 & & 0,1935 & 2079 \\
\hline & 0.5 & 0,2147 & 0,2088 & 0,1813 & 0,2140 & 0,0814 & 0,2771 & 0,1919 & 0,1451 & 0,6012 & 0,4991 & 0,5639 & 0,5460 \\
\hline \multirow[t]{3}{*}{0.5} & 0.2 & 769 & 4 & 465 & 792 & 25 & 99 & 0795 & 0560 & 0,1501 & 1 & 14 & ,1593 \\
\hline & 0.3 & & & & 3 & & & & 67 & & & & \\
\hline & 0.5 & 0,2390 & 0,2255 & 0,1980 & 0,2568 & 0 & 0,3072 & 93 & 0,1537 & 0,6514 & 16 & 0,5935 & 6665 \\
\hline \multirow[t]{3}{*}{0.6} & 0.2 & 54 & 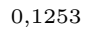 & 0,0530 & 20 & 6 & 0,1965 & 8 & 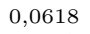 & 24 & 0 & 59 & 58 \\
\hline & 0.3 & & & & & & & & & & & & \\
\hline & 0.5 & 0,2502 & 0,2317 & 0,2148 & 0,2958 & 0,0999 & 0,3124 & 0,2 & 0,1618 & 0,6684 & 51 & 0,6565 & 6675 \\
\hline \multirow[t]{3}{*}{0.7} & 0.2 & 11 & & & & & & & & & & & 247 \\
\hline & 0.3 & & & & & & & & & & & & \\
\hline & 0.5 & 0,2628 & 0,2312 & 0,2455 & 0,3231 & 0,1107 & 0,3371 & 0,2433 & 0,1877 & 0,5978 & 0,4811 & 0,7585 & 0,5771 \\
\hline \multirow[t]{3}{*}{0.8} & 0.2 & 996 & 0 & & 39 & 17 & & & 64 & 68 & & 77 & 364 \\
\hline & 0 & & & & & & & & & & & & \\
\hline & 0.5 & 0,2622 & 0,2359 & 0,2912 & 0,3279 & 0,1204 & 0,3527 & 0,2546 & 0,2032 & 0,5674 & 0,4532 & 0,6933 & 0,5120 \\
\hline \multirow[t]{3}{*}{0.9} & 0.2 & 38 & 0,1438 & 0,1043 & $x$ & 1 & 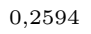 & 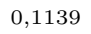 & 0,0919 & 94 & 9 & 41 & 481 \\
\hline & 0 & & & & & & & & & & & & \\
\hline & 0.5 & 6604 & 0,2308 & 0,3140 & 0,3246 & 0,1282 & 0,3739 & 0,2659 & 0,2193 & 0,5294 & 0,4294 & 0,6329 &, 4670 \\
\hline \multirow{4}{*}{$\frac{t_{i}}{0 .}$} & $\Delta_{i}$ & \multicolumn{4}{|c|}{$n_{i}=(30,30,30,30,30)$} & & $=(30,4$ & 60,72, & & & $=(60,6$ & 60,60 & \\
\hline & 0.2 & & & & & & & & & & & & 353 \\
\hline & 0 & & & & & & & & & & & & \\
\hline & 0.5 & 0,2620 & 0,2979 & 0,2928 & 0,3302 & 0,6171 & 0,5706 & 0,4823 & 0,3405 & 0,4669 & 0,3641 & 0,3586 & 0,5564 \\
\hline 0.2 & 0 & 0,0700 & 110 & & 759 & 1 & & 0,0869 & 898 & 57 & 025 & 8 & 08 \\
\hline & & & & & & & & & & & & & \\
\hline & 0.5 & 0,2717 & 0,3291 & 0,3232 & 0,3374 & 0,6627 & 0,5917 & 0,5639 & 0,3908 & 0,4870 & 0,4078 & 0,4051 & 0,5530 \\
\hline 0.3 & 0. & 6684 & 113 & 0,0881 & 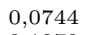 & $P$ & 0,1760 & 0,1192 & 0,1052 & 15 & 0,1205 & 37 & 51 \\
\hline & 0 & & & & & & & & & & & & \\
\hline & 0.5 & 0,2933 & 0,3838 & 0,3854 & 0,3469 & 0,7399 & 0,6543 & 0,7481 & 0,5152 & 0,5461 & 0,5068 & 0,5160 & 0,5682 \\
\hline 0.4 & 0. & 76 & 12 & 0 & 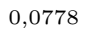 & 0,2098 & 0,2016 & 0,1701 & 0.1581 & 28 & 0,1596 & 4 & 40 \\
\hline & 0 & & & & & & & & & & & & \\
\hline & 0.5 & 0,3367 & 0,4403 & 0,4660 & 0,3741 & 0,8297 & 0,7255 & 0,8882 & 0,7103 & 0,6304 & 0,6553 & 0,7097 & 0,5950 \\
\hline 0.5 & 0 & & & & & & & & & & & & 8 \\
\hline & & & & & & & & & & & & & \\
\hline & 0.5 & 0,3691 & 0,4971 & 0,5577 & 0,4097 & 0,9110 & 0,7760 & 0,8462 & 0,8738 & 0,6932 & 0,7579 & 0,8380 & 0,6293 \\
\hline 0.6 & & & & & & & & & & & & & \\
\hline & & & & & & & & & & & & & \\
\hline & 0.5 & 0,3730 & 0,4827 & 0,5684 & 0,4470 & 0,8338 & 0,7304 & 0,9263 & 0,7489 & 0,6491 & 0,6741 & 0,7713 & 0,6562 \\
\hline 0.7 & & & & & & & & & & & & & \\
\hline & & & & & & & & & & & & & \\
\hline & 0.5 & 0,3608 & 0,4584 & 0,5553 & 0,4906 & 0,7534 & 0,6411 & 0,8004 & 0,5612 & 0,5836 & 0,5357 & 0,6167 & 0,6771 \\
\hline 0.8 & & & & & & & & & & & & & \\
\hline & 0 & & & & & & & & & & & & \\
\hline & 0.5 & 0,3400 & 0,4093 & 0,4896 & 0,5162 & 0,6710 & 0,5906 & 0,6227 & 0,4341 & 0,5267 & 0,4399 & 0,5031 & 0,6866 \\
\hline 0.9 & & & & & & & & & & & & & \\
\hline & 0.3 & & & & & & & & & & & & 801 \\
\hline & 0.5 & 0,3353 & 0,3831 & 0,4766 & 0,5443 & 0,6046 & 0,5680 & 0,5387 & 0,3790 & 0,4987 & 0,3855 & 0,4443 & 0,6925 \\
\hline
\end{tabular}

$\left(\sigma_{1}, \sigma_{2}, \sigma_{3}, \sigma_{4}, \sigma_{5}\right)=(0.2,0.4,0.6,0.8,1.0)$ 
TABle 10. Penalized power of the tests for $k=6$

\begin{tabular}{|c|c|c|c|c|c|c|c|c|c|c|c|c|c|}
\hline & & RGF & MGF & KW & BDM & RGF & MGF & KW & BDM & RGF & MGF & KW & $\mathrm{BDM}$ \\
\hline$t_{i}$ & $\Delta_{i}$ & \multicolumn{4}{|c|}{$n_{i}=(5,8,10,12,14,16)$} & \multicolumn{4}{|c|}{$n_{i}=(10,10,10,10,10,10)$} & \multicolumn{4}{|c|}{$n_{i}=(15,24,30,36,42,48)$} \\
\hline \multirow[t]{3}{*}{0.1} & 0.2 & 0,0736 & 0,1227 & 0,0256 & 0,0538 & 0499 & 0,2314 & 0,0647 & 0,0523 & 0,0975 & 0,1075 & 0,0534 & 0,0760 \\
\hline & 0.3 & 0,0874 & 0,1320 & 0,0359 & 0,0600 & 520 & 0,2362 & 0,0726 & 0,0602 & 0,1406 & 0,1427 & 0,0909 & 0,0907 \\
\hline & 0.5 & 0,1321 & 0,1667 & 0,0838 & 0,1037 & 0,0644 & 0,2584 & 0,1086 & 0,0911 & 0,3320 & 0,2906 & 0,2926 & 0,1959 \\
\hline \multirow[t]{3}{*}{0.2} & 0.2 & 0,0717 & 0,1175 & 0,0241 & 0,0579 & 0,0457 & 0,2146 & 0,0581 & 0,0448 & 0,0929 & 0,1110 & 0,0576 & 0,0719 \\
\hline & 0.3 & 0,0825 & 0,1268 & 0,0357 & 0,0665 & 482 & 0,2218 & 0,0676 & 0,0530 & 1458 & 0,1486 & 0,1048 & 0,0952 \\
\hline & 0.5 & 0,1320 & 0,1648 & 0,0861 & 0,1115 & 0,0615 & 0,2574 & 0,1120 & 0,0912 & 0,3495 & 0,3015 & 0,3291 & 0,2199 \\
\hline \multirow[t]{3}{*}{0.3} & 0.2 & 0,0692 & 0,1141 & 0,0260 & 0,0539 & 0,0403 & 0,2018 & 0,0626 & 0,0440 & 0,0944 & 0,1070 & 0,0581 & 0,0721 \\
\hline & 0.3 & 0,0878 & 0,1247 & 0,0346 & 0,0675 & 426 & 0,2174 & 0,0753 & 0,0543 & 0,1513 & 0,1599 & 0,1116 & 0,1047 \\
\hline & 0.5 & 0,1459 & 0,1660 & 0,0920 & 0,1251 & 0,0595 & 0,2609 & 0,1225 & 0,0921 & 0,3933 & 0,3310 & 0,3734 & 0,2840 \\
\hline \multirow[t]{3}{*}{0.4} & 0.2 & 0,0674 & 0,1152 & 0,0263 & 0,0599 & 0,0375 & 0,1949 & 0,0639 & 0,0444 & 0,1040 & 0,1212 & 0,0568 & 0,0857 \\
\hline & 0.3 & 0,0834 & 0,1291 & 0,0412 & 0,0761 & 427 & 0,2096 & 0,0782 & 0,0543 & 0,1845 & 0,1869 & 0,1177 & 0,1505 \\
\hline & 0.5 & 0,1497 & 0,1770 & 0,0967 & 0,1538 & 0,0638 & 0,2626 & 0,1364 & 0,0969 & 0,4529 & 0,3884 & 0,3587 & 0,3890 \\
\hline \multirow[t]{3}{*}{0.5} & 0.2 & 0,0739 & 0,1189 & 0,0337 & 0,0726 & 0376 & 0,2083 & 0,0734 & 0,0495 & 0,1162 & 0,1296 & 0,0634 & 0,1191 \\
\hline & 0.3 & 228 & 0,1334 & 15 & 0,0994 & 467 & 0,2286 & 0923 & 0,0631 & 1996 & 0,2009 & 0,1366 &, 2132 \\
\hline & 0.5 & 0,1723 & 0,1821 & 0,1137 & 0,1817 & 0,0687 & 0,2953 & 0,1634 & 0,1103 & 0,4946 & 0,4064 & 0,3951 & 0,5240 \\
\hline \multirow[t]{3}{*}{0.6} & 0.2 & 72 & 4 & 0 & 0 & 35 & 0 , & 0,0821 & 0 & 345 & 2 & 6 & 1603 \\
\hline & 0.3 & 11 & & 44 & 2 & & & & & 287 & & & 2686 \\
\hline & 0.5 & 0,1815 & 0,1879 & 0,1332 & 0,2098 & 0,0800 & 0,3098 & 0,1740 & 0,1236 & 0,5127 & 0,4135 & 0,4746 & 0,5561 \\
\hline \multirow[t]{3}{*}{0.7} & 0.2 & 368 & 2 & 3 & 0 & 35 & 0 , & 2 & 4 & 86 & & & 1905 \\
\hline & 0.3 & & & & 20 & & & & & 291 & & & 2857 \\
\hline & 0.5 & 0,1864 & 0,1979 & 0,1555 & 0,2351 & 0,0908 & 0,3357 & 0,1922 & 0,1461 & 0,4748 & 0,3905 & 0,6144 & 0,5041 \\
\hline \multirow[t]{3}{*}{0.8} & 0.2 & 952 & 0,1382 & 568 & 0,1075 & 612 & & 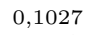 & & 82 & & 52 & ,2091 \\
\hline & 0.3 & & & & & & & & & & & & 2924 \\
\hline & 0.5 & 0,1945 & 0,2024 & 0,1873 & 0,2477 & 0,0981 & 0,3408 & 0,2015 & 0,1614 & 0,4539 & 0,3708 & 0,6393 & 0,4464 \\
\hline \multirow[t]{3}{*}{0.9} & 0.2 & 0,0995 & 446 & 81 & 0,1174 & 678 & 0 & 6 & $z$ & 649 & 14 & 32 & 0,2206 \\
\hline & 0.3 & & & & & & & & & & & & 2949 \\
\hline & 0.5 & 0,2000 & 0,2084 & 0,2094 & 0,2580 & 1026 & 0,3479 & 0,2059 & 0,1735 & ,4409 & 0,3637 & 0,6057 & 0,4070 \\
\hline \multirow{4}{*}{$\frac{t_{i}}{0.1}$} & $\Delta_{i}$ & $n_{i}$ & $(30,30$, & $30,30,30$ & 30) & $n_{i}$ & $=(30,48$, & $60,72,84$, & 96) & $n_{i}$ & $=(60,60$, & $50,60,60$ & 60) \\
\hline & 0.2 & 684 & & 0 & 0 , & 254 & & & & 938 & 34 & & 0,1457 \\
\hline & 0. & & & & & & & & & & & & 981 \\
\hline & 0.5 & 0,1618 & 0,2135 & 0,1997 & 0,2260 & 0,5181 & 0,4718 & 0,4060 & 0,2633 & 0,3349 & 0,2673 & 0,2729 & 0,4271 \\
\hline \multirow[t]{3}{*}{0.2} & 0.2 & 619 & 0 & 0 & 0,0710 & 30 & 0,1 & 0,0 & & 30 & 66 & 8 & 233 \\
\hline & 0 . & & & & & & & & & & & & 824 \\
\hline & 0.5 & 0,1678 & 0,2336 & 0,2176 & 0,2202 & 0,5612 & 0,4995 & 0,4892 & 0,3005 & 0,3512 & 0,3016 & 0,3089 & 0,4203 \\
\hline 0.3 & 0.2 & 595 & 0 & 0 & 0,0663 & 23 & 0 & 0,0827 & $p$ & 00 & 20 & 0 & 027 \\
\hline & 0. & & & & & & & & & & & & 704 \\
\hline & 0.5 & 0,1784 & 0,2677 & 0,2623 & 0,2301 & 0,6448 & 0,5573 & 0,6588 & 0,3937 & 0,3907 & 0,3737 & 0,3902 & 0,4316 \\
\hline 0.4 & 0.2 & 624 & 0 & 0 & $x$ & 0,1565 & 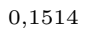 & 0,0953 & J & 53 & 18 & 64 & 0,0948 \\
\hline & 0 . & & & & & & & & & & & & \\
\hline & 0.5 & 0,2104 & 0,3140 & 0,3263 & 0,2547 & 0,7522 & 0,6302 & 0,6649 & 0,6115 & 0,4691 & 0,5126 & 0,5476 & 0,4585 \\
\hline 0.5 & 0.2 & 96 & 0 & 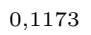 & 0,0798 & 0,1910 & 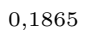 & 0,1279 & 0,1895 & 1 & 0,1526 & 0 & 175 \\
\hline & 0 . & & & & & & & & & & & & \\
\hline & 0.5 & 0,2430 & 0,3645 & 0,4024 & 0,2915 & 0,8239 & 0,6896 & 0,6850 & 0,8038 & 0,5196 & 0,5911 & 0,6986 & 0,5013 \\
\hline 0.6 & 0. & 0 & & 0 & 0,1048 & 0,2093 & & 0,2073 & 0,2625 & 4 & 4 & 3 & 98 \\
\hline & & & & & & & & & & & & & 966 \\
\hline & 0.5 & 0,2559 & 0,3719 & 0,4410 & 0,3393 & 0,7823 & 0,6597 & 0,7794 & 0,7079 & 0,5069 & 0,5445 & 0,6771 & 0,5613 \\
\hline 0.7 & 0. & 0 & & & & & & & & & & & 0,2577 \\
\hline & & & & & & & & & & & & & 0,3827 \\
\hline & 0.5 & 0,2643 & 0,3746 & 0,4497 & 0,3975 & 0,6820 & 0,5861 & 0,8431 & 0,5170 & 0,4630 & 0,4407 & 0,5548 & 0,6061 \\
\hline 0.8 & & & & & & & & & & & & & \\
\hline & 0.3 & & & & & & & & & & & & 0,4599 \\
\hline & 0.5 & 0,2618 & 0,3429 & 0,4207 & 0,4394 & 0,6113 & 0,5257 & 0,6661 & 0,4022 & 0,4226 & 0,3572 & 0,4557 & 0,6384 \\
\hline 0.9 & & & & & & & & & & & & & \\
\hline & 0.3 & & & & & & & & & & & 0,3245 & 0,5093 \\
\hline & 0.5 & 0,2604 & 0,3231 & 0,4101 & 0,4739 & 0,5580 & 0,4979 & 0,5628 & 0,3504 & 0,4069 & 0,3181 & 0,4066 & 0,6568 \\
\hline
\end{tabular}


TABle 11. Penalized power of the tests for $k=7$

\begin{tabular}{|c|c|c|c|c|c|c|c|c|c|c|c|c|c|}
\hline & & RGF & MGF & KW & BDM & RGF & MGF & $\mathrm{KW}$ & BDM & RGF & MGF & $\mathrm{KW}$ & BDM \\
\hline$t_{i}$ & $\Delta_{i}$ & \multicolumn{4}{|c|}{$n_{i}=(4,6,8,10,12,14,16)$} & \multicolumn{4}{|c|}{$n_{i}=(10,10,10,10,10,10,10)$} & \multicolumn{4}{|c|}{$n_{i}=(12,18,24,30,36,42,48)$} \\
\hline \multirow[t]{3}{*}{0.1} & 0.2 & 787 & 0,1301 & 0,0310 & & & & 0,0668 & & & 0,1110 & 0,0494 & 0,0828 \\
\hline & . & 853 & 6 & 4 & 0,0635 & 0531 & & 0,0704 & & 0,1196 & & 0,0712 & 0,0896 \\
\hline & & 0,1138 & 0,1564 & 0,0754 & 0,0905 & 0,0608 & 0,2784 & 0,0937 & 0,0765 & 0,2299 & 0,2192 & 0,1899 & 0,1465 \\
\hline \multirow[t]{3}{*}{0.2} & 0.2 & 0,0782 & 0,1282 & 0,0289 & 0,0595 & 0,0469 & 0,2502 & 0,0595 & 0,0450 & 0,0824 & 0,1047 & 0,0466 & 0,0680 \\
\hline & & & & 0382 & 0,0647 & 0481 & & 27 & 0,0468 & 0,1101 & & 0,0711 & 0812 \\
\hline & 0.5 & 0,1131 & 0,1545 & 0,0704 & 0,0918 & 0,0559 & 0,2762 & 0,0878 & 0,0677 & 0,2403 & 0,2297 & 0,2148 & 0,1618 \\
\hline \multirow[t]{3}{*}{0.3} & 0.2 & 0,0711 & 0,1190 & 0,0264 & 0,0567 & 0,0422 & 0,2305 & 0,0628 & 0,0444 & 0,0812 & 0,1021 & 0,0435 & 0,0669 \\
\hline & 0.3 & & 3 & 2 & 0 & 0432 & & 0,0691 & 0,0501 & 0,1128 & & 0,0778 & 0874 \\
\hline & 0.5 & 0,1136 & 0,1549 & 0,0635 & 0,0971 & 0,0543 & 0,2617 & 0,0989 & 0,0716 & 0,2593 & 0,2431 & 0,2431 & 0,1945 \\
\hline \multirow[t]{3}{*}{0.4} & 0.2 & 0,0656 & 0,1181 & 0,0247 & 0,0566 & 0,0371 & 0,2178 & 0,0604 & 0,0396 & 0,0802 & 0,1010 & 0,0429 & 0,0729 \\
\hline & 0.3 & 760 & 5 & 352 & 0,0687 & 0400 & & 89 & 0,0464 & 0,1281 & & 0,0803 &, 1136 \\
\hline & 0.5 & 0,1209 & 0,1571 & 0,0748 & 0,1177 & 0,0513 & 0,2637 & 0,1111 & 0,0752 & 0,3053 & 0,2791 & 0,2462 & 0,2719 \\
\hline \multirow[t]{3}{*}{0.5} & 0.2 & 0,0657 & 1156 & 0,0275 & 0,0608 & 0398 & 0,2204 & 0,0718 & 0,0458 & 0,0850 & 1087 & 0,0488 &, 0906 \\
\hline & 0.3 & 791 & & 0391 & 817 & & & & 0,0550 & 0,1390 & & 0,0984 & 1543 \\
\hline & 0.5 & 0,1253 & 0,1597 & 0,0855 & 0,1334 & 0,0587 & 0,2874 & 0,1294 & 0,0839 & 0,3447 & 0,3055 & 0,2819 & 0,3791 \\
\hline \multirow[t]{3}{*}{0.6} & 0.2 & 2 & 1 & 0,0315 & 0,0732 & 2 & 7 & 1 & 33 & 5 & 6 & 38 & 318 \\
\hline & 0.3 & 866 & & & 3 & & & & 70 & 59 & & & 2034 \\
\hline & 0.5 & 0,1421 & 0,1706 & 0,1020 & 0,1613 & 0,0669 & 0,3018 & 0,1458 & 0,1024 & 0,3575 & 0,3106 & 0,3482 & 0,4184 \\
\hline \multirow[t]{3}{*}{0.7} & 0.2 & 827 & 5 & 2 & 0,0885 & 3 & ,2657 & 3 & 0655 & 4 & 0 & 99 & 34 \\
\hline & 0.3 & 956 & & & & & & & 83 & 0 & & & \\
\hline & 0.5 & 0,1482 & 0,1740 & 0,1163 & 0,1813 & 0,0779 & 0,3258 & 0,1644 & 0,1218 & 0,3505 & 0,2987 & 0,4591 & 0,3927 \\
\hline \multirow[t]{3}{*}{0.8} & 0.2 & 907 & & -5 & (2006 & & & & 078 & 0,13 & & 5 & \\
\hline & 0 & & & & & & & & & & & & \\
\hline & 0.5 & 0,1564 & 0,1830 & 0,1354 & 0,2005 & 0,0865 & 0,3338 & 0,1653 & 0,1332 & 0,3492 & 0,2980 & 0,5125 & 0,3786 \\
\hline \multirow[t]{3}{*}{0.9} & 0. & 996 & & & & & & & & 0,1488 & & & \\
\hline & 0 & & & & & & & & & & & & \\
\hline & 0.5 & 0,1642 & 0,1849 & 0,1599 & 0,2066 & 0,0950 & 0,3444 & 0,1728 & 0,1481 & 0,3442 & 0,2981 & 0,4955 & 0,3616 \\
\hline \multirow{4}{*}{$\begin{array}{l}t_{i} \\
0.1\end{array}$} & $\Delta_{i}$ & $n_{i}=$ & $30,30,3$ & 0,30 & 30) & $n_{i}=$ & 4,36 , & 60,72 & 96) & $n_{i}=$ & $(60,60$, & 60,60 & $0,60)$ \\
\hline & 0.2 & 0,0718 & & & & & & & & 0,0860 & & & \\
\hline & 0 & & & & & & & & & 0,1 & & & \\
\hline & 0.5 & 0,1311 & 0,1847 & 0,1676 & 0,1844 & 0,3950 & 0,3655 & 0,2831 & 0,2038 & 0,2312 & 0,1933 & 0,2101 & 0,3366 \\
\hline \multirow[t]{3}{*}{0.2} & 0.2 & & & & & & & & & & & & \\
\hline & 0 & & & & & & & & & 0,1 & & & \\
\hline & 0.5 & 0,1287 & 0,2019 & 0,1750 & 0,1759 & 0,4344 & 0,3900 & 0,3425 & 0,2256 & 0,2347 & 0,2144 & 0,2268 & 0,3159 \\
\hline 0.3 & 0.2 & & & & & & & & & & & & \\
\hline & 0 & & & & & & & & & & & & \\
\hline & 0.5 & 0,1365 & 0,2237 & 0,2046 & 0,1772 & 0,4921 & 0,4326 & 0,4733 & 0,2940 & 0,2569 & 0,2650 & 0,2773 & 0,3063 \\
\hline 0.4 & 0 . & 0 & & & & & & & & & & & 2 \\
\hline & & & & & & & & & & & & & \\
\hline & 0.5 & 0,1536 & 0,2606 & 0,2583 & 0,1945 & 0,5690 & 0,4969 & 0,5354 & 0,4592 & 0,3212 & 0,3708 & 0,4051 & 0,3335 \\
\hline 0.5 & 0 . & 0 & & 0,1071 & 0 & 0 & 385 & 7 & 0,1442 & 0,0 & 5 & 3 & 932 \\
\hline & & & & & & & & & & & & & \\
\hline & 0.5 & 0,1713 & 0,2951 & 0,3098 & 0,2151 & 0,6463 & 0,5553 & 0,5710 & 0,6773 & 0,3770 & 0,4524 & 0,5666 & 0,3906 \\
\hline 0.6 & 0. & 0 & 0,1431 & 0,1238 & 0 & 0,1598 & 0,1633 & 9 & 0,2093 & 0 & 3 & 7 & 51 \\
\hline & & & & & & & & & & & & & \\
\hline & 0.5 & 0,1883 & 0,3071 & 0,3474 & 0,2642 & 0,6557 & 0,5518 & 0,7011 & 0,6345 & 0,3830 & 0,4366 & 0,5722 & 0,4608 \\
\hline 0.7 & 0. & & & & & & & & & 0,1238 & & 3 & 88 \\
\hline & & & & & & & & & & & & & \\
\hline & 0.5 & 0,1898 & 0,3028 & 0,3545 & 0,3084 & 0,5963 & 0,5031 & 0,7442 & 0,4809 & 0,3647 & 0,3691 & 0,4893 & 0,5344 \\
\hline 0.8 & 0 . & & & & & & & & & & & & \\
\hline & & & & & & & & & & & & & \\
\hline & 0.5 & 0,1960 & 0,2849 & 0,3479 & 0,3564 & 0,5242 & 0,4555 & 0,5764 & 0,3808 & 0,3429 & 0,3029 & 0,4082 & 0,5924 \\
\hline 0.9 & 0 & & & & & & & & & & & & \\
\hline & 0 & & & & & & & & & & & & \\
\hline & 0.5 & 0,2021 & 0,2707 & 0,3378 & 0,3944 & 0,4829 & 0,4303 & 0,4982 & 0,3282 & 0,3395 & 0,2752 & 0,3799 & 0,6265 \\
\hline
\end{tabular}

$\left(\sigma_{1}, \sigma_{2}, \sigma_{3}, \sigma_{4}, \sigma_{5}, \sigma_{6}, \sigma_{7}\right)=(0.2,0.4,0.6,0.8,1.0,1.2,1.4)$ 
Authors Contribution Statements The authors contributed equally. All authors read and approved the final copy of the paper.

Declaration of Competing Interests The authors declare that they have no known competing financial interests or personal relationships that could have appeared to influence the work reported in this paper.

Acknowledgement This study is supported by the Eskisehir Technical University Scientific Research Projects Commission under grant No. 20 DRP047.

\section{REFERENCES}

[1] Bradley, J. V., Robustness?, British Journal of Mathematical and Statistical Psychology, 31 (1978), 144-152. https://doi.org/10.1111/j.2044-8317.1978.tb00581.x

[2] Brunner, E., Dette, H., Munk, A., Box-type approximations in nonparametric factorial designs, Journal of the American Statistical Association, 92 (1997), 1494-150. https://doi.org/10.1080/01621459.1997.10473671

[3] Cavus, M., Yazici, B., Sezer, A., Modified tests for comparison of group means under heteroskedasticity and non-normality caused by outlier(s), Hacettepe Journal of Mathematics and Statistics, 46 (2017), 493-510. https://doi.org/10.15672/HJMS.2017.417

[4] Cavus, M., Yazici, B., Sezer, A., Analyzing regional export data by the modified generalized F-test, International Journal of Economic and Administrative Studies, (2018), 541-552. https://doi.org/10.18092/ulikidince.348070

[5] Cavus, M., Yazici, B., Testing the equality of normal distributed and independent groups' means under unequal variances by doex package, The $R$ Journal, 12 (2020), 134-154. https://doi.org/10.32614/RJ-2021-008

[6] Cavus, M., Yazici, B., Sezer, A., Penalized power approach to compare the power of the tests when Type I error probabilities are different, Communications in Statistics - Simulation and Computation, 50 (7) (2021), 1912-1926. https://doi.org/10.1080/03610918.2019.1588310

[7] Cribbie, R. A., Fiksenbaum, L., Keselman, H. J., Wilcox, R. R., Effect of non-normality on test statistics for one-way independent group designs, British Journal of Mathematical and Statistical Psychology, 65 (2012), 56-73. https://doi.org/10.1111/j.2044-8317.2011.02014.x

[8] Cribbie, R. A., Wilcox, R. R., Bewell, C., and Keselman, H. J., Tests for treatment group equality when data are nonnormal and heteroscedastic, Journal of Modern Applied Statistical Methods, 6 (2007), 117-132. https://doi.org/10.22237/jmasm/1177992660

[9] Fagerland, M. W., Sandvik, L., The Wilcoxon-Mann-Whitney test under scrutiny, Statistics in Medicine, 28 (2009), 1487-1497. https://doi.org/10.1002/sim.3561

[10] Huber, P. J., Robust estimation of a location parameter, Annals of Mathematical Statistics, 35 (1964), 73-101. https://doi.org/10.1214/aoms/1177703732

[11] Huber, P. J. Robust Statistics. New York: John Wiley and Sons, 2013. ISBN: 978-0-47012990-6.

[12] Karagoz, D., Saracbasi, T., Robust Brown-Forsythe and robust modified Brown-Forsythe ANOVA tests under heteroscedasticity for contaminated Weibull distribution, Revista Colombiana de Estadistica, 39 (2016), 17-32. https://doi.org/10.15446/rce.v39n1.55135

[13] Keselman, H.J., Wilcox, R. R., Othman, A. R., Fradette, K., Trimming, transforming statistics and bootstrapping: circumventing the biasing effects of heteroscedasticity and non-normality, Journal of Modern Applied Statistical Methods, 1 (2002), 288-309. https://doi.org/10.22237/jmasm/1036109820 
[14] Kruskal, W. H., Wallis, W. A., Use of ranks in one-criterion variance analysis, Journal of the American Statistical Association, 47 (1952), 583-621. https://doi.org/10.2307/2280779

[15] Kulinskaya, E., Dollinger, M. B., Robust weighted one-way ANOVA: improved approximation and efficiency, Journal of Statistical Planning and Inference, 137 (2007), 462-472. https://doi.org/10.1016/j.jspi.2006.01.008.

[16] Luh, W. M., Guo, J. H., A powerful transformation trimmed mean method for one-way fixed effects ANOVA model under non-normality and inequality of variances, British Journal of Mathematical and Statistical Psychology, 52 (1999), 303-320. https://doi.org/10.1348/000711099159125

[17] Luh, W. M., Guo, J. H., Heteroscedastic test statistics for one-way analysis of variance: the trimmed means and Hall's transformation conjunction. The Journal of Experimental Education, 74 (2005), 75-100. https://doi.org/10.3200/JEXE.74.1.75-100.

[18] Ochuko, T. K., Abdullah, S., Zain, Z. B., Yahaya, S. S. S., The modification and evaluation of the Alexander-Govern tests in terms of power, Modern Applied Science, 9 (2015), 1-21. https://doi.org/10.5539/mas.v9n13p1.

[19] Oshima, T., Algina, J., Type I error rates for James's second-order test and Wilcox's HM test under heteroscedasticity and non-normality, British Journal of Mathematical and Statistical Psychology, 45 (1992), 255-263. https://doi.org/10.1111/j.2044-8317.1992.tb00991.x

[20] Ozdemir, A. F., Wilcox, R. R. Yildiztepe, E., Comparing j independent groups with a method based on trimmed mean, Communications in Statistics-Simulation and Computation, 47 (2018), 852-863. https://doi.org/10.1080/03610918.2017.1295152

[21] Weerahandi, S., ANOVA under unequal error variances. Biometric, 51 (1995), 589-599. https://doi.org/10.2307/2532947.

[22] Yazici, B., Cavus, M., A comparative study of computation approaches of the generalized F-test. Journal of Applied Statistics, 48 (2021), 2906-2919. https://doi.org/10.1080/02664763.2021.1939660

[23] Yusof, Z., Abdullah, S., Yahaya, S. S. S., Comparing the performance of modified Ft statistic with ANOVA and Kruskal Wallis test. Applied Mathematics and Information Sciences, 7 (2013), 403-408. https://doi.org/10.12785/amis/072L04

[24] Zhu, D., Zinde-Walsh, V., Properties of estimation of asymmetric exponential power distribution. Journal of Econometrics, 148 (2009), 89-99. https://doi.org/10.1016/j.jeconom.2008.09.038 